\title{
Dark matter direct detection signals inferred from a cosmological N-body simulation with baryons
}

\author{
Ling, F S ; Nezri, E ; Athanassoula, E ; Teyssier, R
}

\begin{abstract}
We extract at redshift $\mathrm{z}=0$ a Milky Way sized object including gas, stars and dark matter (DM) from a recent, high-resolution cosmological N-body simulation with baryons. Its resolution is sufficient to witness the formation of a rotating disk and bulge at the center of the halo potential, therefore providing a realistic description of the birth and the evolution of galactic structures in the $\Lambda \mathrm{CDM}$ cosmology paradigm. The phase-space structure of the central galaxy reveals that, throughout a thick region, the dark halo is co-rotating on average with the stellar disk. At the Earth's location, the rotating component, sometimes called dark disk in the literature, is characterized by a minimum lag velocity vlag simeq $75 \mathrm{~km} / \mathrm{s}$, in which case it contributes to around $25 \%$ of the total DM local density, whose value is $\mathrm{DM}$ simeq $0.37 \mathrm{GeV} / \mathrm{cm} 3$. The velocity distributions also show strong deviations from pure Gaussian and Maxwellian distributions, with a sharper drop of the high velocity tail. We give a detailed study of the impact of these features on the predictions for DM signals in direct detection experiments. In particular, the question of whether the modulation signal observed by DAMA is or is not excluded by limits set by other experiments (CDMS, XENON and CRESST...) is re-analyzed and compared to the case of a standard Maxwellian halo. We consider spin-independent interactions for both the elastic and the inelastic scattering scenarios. For the first time, we calculate the allowed regions for DAMA and the exclusion limits of other null experiments directly from the velocity distributions found in the simulation. We then compare these results with the predictions of various analytical distributions. We find that the compatibility between DAMA and the other experiments is improved. In the elastic scenario, the DAMA modulation signal is slightly enhanced in the so-called channeling region, as a result of several effects that include a departure from a Maxwellian distribution and anisotropies in the velocity dispersions due to the dark disk. For the inelastic scenario, the improvement of the fit is mainly attributable to the departure from a Maxwellian distribution at high velocity. It is correctly modeled by a generalized Maxwellian distribution with a parameter simeq 1.95 , or by a Tsallis distribution with q simeq 0.75 .
\end{abstract}

DOI: https://doi.org/10.1088/1475-7516/2010/02/012

Posted at the Zurich Open Repository and Archive, University of Zurich

ZORA URL: https://doi.org/10.5167/uzh-34435

Journal Article

Accepted Version

Originally published at:

Ling, F S; Nezri, E; Athanassoula, E; Teyssier, R (2010). Dark matter direct detection signals inferred from a cosmological N-body simulation with baryons. Journal of Cosmology and Astroparticle Physics, 2010(2):12.

DOI: https://doi.org/10.1088/1475-7516/2010/02/012 
ULB-TH/09-30

\title{
Dark Matter Direct Detection Signals inferred from a Cosmological N-body Simulation with Baryons
}

\author{
F.-S. Ling ${ }^{1}$, E. Nezri² ${ }^{2}$ E. Athanassoula ${ }^{2}$, R. Teyssier ${ }^{3}$ \\ ${ }^{1}$ Service de Physique Théorique, Université Libre de Bruxelles, \\ CP225, Bld du Triomphe, 1050 Brussels, Belgium \\ ${ }^{2}$ Laboratoire d'Astrophysique de Marseille, \\ Observatoire Astronomique de Marseille Provence \\ CNRS/Université de Provence \\ 38 rue Joliot Curie, 13388 Marseille, France \\ ${ }^{3}$ IRFU, CEA Saclay \\ L'Orme des merisiers 91191 Gif-sur-Yvette, France
}

\begin{abstract}
We extract at redshift $z=0$ a Milky Way sized object including gas, stars and dark matter (DM) from a recent, high-resolution cosmological N-body simulation with baryons. Its resolution is sufficient to witness the formation of a rotating disk and bulge at the center of the halo potential, therefore providing a realistic description of the birth and the evolution of galactic structures in the $\Lambda \mathrm{CDM}$ cosmology paradigm.

The phase-space structure of the central galactic halo reveals the presence of a dark disk component, that is co-rotating with the stellar disk. At the Earth's location, the rotating component is characterized by an average lag velocity $v_{\text {lag }} \simeq 75 \mathrm{~km} / \mathrm{s}$, and contributes to around $25 \%$ of the total DM local density, whose value is $\rho_{D M} \simeq 0.37 \mathrm{GeV} / \mathrm{cm}^{3}$. The velocity distributions also show strong deviations from pure Gaussian and Maxwellian distributions, with a sharper drop of the high velocity tail.

We give a detailed study of the impact of these features on the predictions for DM signals in direct detection experiments. In particular, the question of whether the modulation signal observed by DAMA is or is not excluded by limits set by other experiments (CDMS, XENON and CRESST...) is re-analyzed and compared to the case of a standard Maxwellian halo. We consider spin-independent interactions for both the elastic and the inelastic scattering scenarios. For the first time, we calculate the allowed regions for DAMA and the exclusion limits of other null experiments directly from the velocity distributions found in the simulation. We then compare these results with the predictions of various analytical distributions.

We find that the compatibility between DAMA and the other experiments is improved. In the elastic scenario, the DAMA modulation signal is slightly enhanced in the so-called channeling region, as a result of several effects that include a departure from a Maxwellian distribution and anisotropies in the velocity dispersions due to the dark disk. For the inelastic scenario, the improvement of the fit is mainly attributable to the departure from a Maxwellian distribution at high velocity. It is correctly modeled by a generalized Maxwellian distribution with a parameter $\alpha=1.95$.
\end{abstract}




\section{Introduction}

Nowadays, the hypothesis of dark matter (DM) is considered as the most plausible explanation for various observations from galactic to cosmological scales. Flat rotation curves in spiral galaxies [1, $2]$, velocity dispersions in galaxy clusters [3, 4], gravitational lensing mass reconstructions [5, 6, 7], hierarchical patterns in large scale structures $[8,9]$ and cosmic microwave background anisotropies [10] all point towards the existence of a new neutral, non baryonic, cold (i.e. with low thermal velocities) and weakly interacting massive particle (WIMP) in the universe.

Theories beyond the Standard Model of particle physics [11, 12, 13] provide many candidates for DM particles (see for example Refs. [14, 15, 16, 17]). However, its existence will not be proved and its properties will not be determined until a clear identification is made through signals in indirect or direct detection experiments or in particle accelerators. Recently, several astrophysical excesses have been reported [18, 19, 20,21], and associated with a DM signal in more or less exotic scenarios (see, among many others, Refs. [22, 23, 24, 25, 26, 27]). However, up to now, none of them requires $\mathrm{DM}$ as an irrefutable explanation. The INTEGRAL $511 \mathrm{keV}$ line $\gamma$-ray signal [18] can be explained by the emission of low mass X-ray binaries [28]. The EGRET diffuse $\gamma$-ray $\mathrm{GeV}$ anomaly [19] was most likely due to an error in the energy calibration [29], and has since indeed been ruled out by the FERMI instrument [30]. The positron and electron peak seen by ATIC [21] has been flattened by HESS [31] and FERMI [32] measurements. The positron fraction rise observed by PAMELA [20], although striking, can be accommodated in standard astrophysical scenarios with pulsars [33, 34], or with an inhomogeneous distribution of supernovae remnants [35].

Direct detection provides a unique opportunity to disentangle DM signals from other astrophysical processes. The idea is to directly measure the energy deposited in a low noise detector during the collision between a DM particle and a detector nucleus. Several experiments have been designed for this aim [36, 37, 38, 39, 40]. Up to now, all of them are compatible with null results, except the DAMA/LIBRA collaboration which claims to have observed a signal with an annual modulation characteristic of an DM signal [41]. This modulation is induced by the Earth rotation around the Sun, and is presented as a signature hard to reproduce by background events. The DAMA result is still quite controversial because it leads to tensions with other experiment measurements (for a recent status, see Refs. [42, 43, 44, 45]).

Predicting direct detection signals in any particle physics model requires astrophysical assumptions in the form of a value for the local DM density as well as velocity distributions in the solar neighborhood. Usually, the local DM density is taken as $\rho_{D M}=0.3 \mathrm{GeV} / \mathrm{cm}^{3}=0.079 \mathrm{M}_{\mathrm{Sun}} / \mathrm{pc}^{3}$, a value which is supported by the Milky-Way rotation curve [46, 47], and stars spectrophotometric data [48]. A recent determination which takes numerous dynamical observables into account gives a larger value, $\rho_{D M}=0.39 \mathrm{GeV} / \mathrm{cm}^{3}$ [49]. However, it should be mentioned that determinations based on mass models only give average values. A local peak in the DM density, although improbable without a dynamical reason [50], is in principle not excluded by very local bounds based on planetary data [51, 52]. Another common over-simplified assumption is to take the velocity distribution as isotropic and Maxwellian. Incomplete virialization, streams and interaction with the stellar disk could contribute to create anisotropies or a non Maxwellian spectrum. The consequences for direct detection of some of these departures from the standard halo case have been analyzed by several authors $[53,54,55,56,57$, $58,59,60]$.

Numerical simulations have been used for decades as a powerful virtual laboratory to explore complex dynamical systems. On cosmological scales, early studies enabled to assess the role of DM in the hierarchical structure formation sheme [61, 62]. Recent techniques [63,64] as well as improving computing capabilities have brought the possibility to extract galactic DM halos with tremendous resolution. The so-called Via Lactea simulation [65] or the Aquarius project [66] have resolved a Milky-Way sized galactic halo with more than a billion particles. It appears that numerous sub-halos are present, anisotropies and deviations from standard Maxwellian velocity distributions are also found and lead to somewhat different signatures in direct detection experiments $[67,43,60]$. Despite their impressive resolution, these DM only simulations fail as a realistic description of a galactic halo for the simple reason that they totally neglect the baryonic components (stars and gas in the galactic disk and bulge), although these are known to dominate the dynamics near the galactic center. This shortcoming is being 
fixed by very recent cosmological simulations which include baryons $[68,69]$. It appears that the DM spatial distribution is neither spherical, or triaxial, but has a thick oblate shape around the galactic disk, in what some authors have called a dark disk [70, 71], that is co-rotating with the galactic stellar disk. These particular characteristics lead to a potential enhancement of the direct detection signal at low recoil energies [71], although there is an important spread in the predictions, depending on the particular merger history for each galaxy.

In this paper, a recent and advanced cosmological simulation with baryons is used as a realistic framework to extract detailed predictions for direct detection. The paper is organized as follows. In Sec. 2, the simulation is presented and described. Velocity distributions in the solar neighborhood are extracted, analyzed and compared with standard simplified assumptions. The presence of a corotating dark disk is also discussed. In Sec. 3, after a brief overview of the event rate formalism and the experimental status, the direct detection predictions are given for both the elastic and the inelastic scenarios. Finally, our results are summarized in Sec. 4.

\section{A cosmological simulation with baryons}

Although galaxy formation is far from being completely understood, both theory and observation have made significant progresses in the recent years. Based on first principles, it is now possible to perform simulations of Milky-Way-like galaxies in reasonable agreement with observed galaxies of similar circular velocities $[72,73,74]$. Some long-lasting problems seem however to remain, especially for massive galaxies like our own: the simulated bulge seems systematically too massive (sometimes referred to as the angular momentum problem), and the total baryon fraction too large (the overcooling problem). Following previous work initiated by Bruch et al. [71], we nevertheless would like to use a Milky-Way-like galaxy simulation including both dark matter and baryons dynamics to infer the expected signal in dark matter direct detection experiments.

\section{$2.1 \quad$ Features}

The simulation was performed using the cosmological Adaptive Mesh Refinement (AMR) code RAMSES [75]. We follow the evolution of 2 collisionless fluids, namely dark matter and stars, coupled through gravity to the dissipative baryonic component. Gas dynamics is described using a secondorder unsplit Godunov scheme. Gas is allowed to cool radiatively, based on a standard equilibrium photo-ionised mixture of Hydrogen and Helium [76], taken into account the excess cooling due to metals [77]. Star formation is implemented using a standard Schmidt law, spawning stars as a Poisson random process [78]. Our rather standard recipe for supernovae feedback and the associated chemical enrichment is described in Ref. [79].

We used the so-called "zoom-in" simulation technique, for which we identify a candidate halo using a low resolution, dark matter only simulation, and then re-simulate it at higher resolution, degrading both spatial and mass resolutions as we move further away from the central region. We checked that our final halo is not contaminated by high mass, low resolution dark matter particles. Our effective resolution in the initial conditions corresponds to a Cartesian grid of $1024^{3}$ elements covering our $20 \mathrm{Mpc} / h$ periodic box. The corresponding dark matter particle mass is therefore $7.46 \times 10^{5}$ solar masses, given we used the following cosmological model $\Omega_{m}=0.3, \Omega_{\Lambda}=0.7, \Omega_{b}=0.045, H_{0}=70 \mathrm{~km} / \mathrm{s} / \mathrm{Mpc}$. The initial Gaussian random field was generated according to the Eisenstein and Hu (1999) $\Lambda$ CDM model [80] for the transfer function.

At redshift $z=0$, the complete simulation volume contains 8,376,152 dark matter particles of various masses, 11,702,370 star particles and 19,677,225 AMR cells. We have fixed the spatial resolution to be $200 \mathrm{pc}$ in physical units, which ensures that the vertical scale height of the stellar disc is barely resolved. The final halo Virial radius $R_{v i r}{ }^{1}$ is found to be $264 \mathrm{kpc}$. It contains $N_{D M}=842,768$ dark matter particles corresponding to a halo mass $M_{h}=6.310^{11} \mathrm{M}_{\text {sun }}$. Within the central $60 \mathrm{kpc}$, we have $N_{*}=5,416,865$ stars particles (within $15 \mathrm{kpc}$, this number goes down to $N_{*}=4,927,325$ particles).

\footnotetext{
${ }^{1}$ The Virial radius $R_{v i r}$ is defined as a function of the cosmological matter density $\rho_{m}$ by $\frac{M\left(R<R_{v i r}\right)}{4 / 3 \pi R_{v i r}^{3}}=200 \rho_{m}$
} 
As discussed above, our central galaxy is slightly too concentrated, with a bulge mass of the order of $4 \times 10^{10} \mathrm{M}_{\text {sun }}$ and a similar disc mass, while the most recent observational constraints in the Milky Way suggest a bulge mass of $2 \times 10^{10} \mathrm{M}_{\text {sun }}$ for a disc mass of $6 \times 10^{10} \mathrm{M}_{\text {sun }}$ [47]. Although the total mass in stars of our simulated agrees quite well with observations, we stress that we have considered here a rather low mass halo model $\left(M_{v i r} \simeq 6 \times 10^{11} \mathrm{M}_{\text {sun }}\right)$, resulting in a total baryon fraction in the galaxy close to the universal value of $15 \%$.

The central dark halo, stars and gas are shown respectively on figure 2 and 3. Figure 2 shows the dark matter distribution. As expected, it is centrally concentrated and has a considerable number of sub-halos. Figure 3 shows the distribution of the stars and the gas. Both display a very thin disk of a radial extent of roughly $10 \mathrm{kpc}$ and a clear spiral structure. For the stellar population, this is mainly seen in the outer parts of the disc, while for the gas it can be traced to a region very near the center. In both cases it is two-armed, but has clear asymmetries. Secondary branchings of the arms, coupled to the main arms result in a ring-like shape in the gas distribution.

Note also that there is an important central concentration in both distributions. This is of short extent, both in the equatorial plane and perpendicular to it. The latter property, as well its rich gaseous content which has roughly the same extent as the stellar distribution, exclude the possibility that it is a classical bulge and argue strongly that is a discy bulge [81]. Further investigations of this point is nevertheless necessary.

In the stellar distribution the main galaxy is surrounded by a number of small condensations, big enough to be considered as small satellite galaxies. In the gaseous distribution, however, only one of these condensations can be seen. This means that the remaining condensations have no gaseous counterpart; the gas having been expelled either by ram pressure stripping, or by a central supernova, or a combination of both. The clear filamentary structures in the gaseous edge-on plot outline past satellite trajectories.

\section{$2.2 \quad$ Velocity distributions}

In this section, we extract and analyze the velocity distributions given by our N-body simulation, and compare them with the usual Gaussian/Maxwellian assumption. As the goal of this study is to calculate direct detection predictions with a realistic DM halo, the emphasis will be put on the local phase-space structure, in the solar neighborhood.

The Sun being located at a distance $R_{0} \simeq 8 \mathrm{kpc}$ from the galactic center, we first select all DM particles in the spherical shell $7 \mathrm{kpc}<R<9 \mathrm{kpc}$. The number of particles in the selection is $N_{\text {shell }}=16,545$. Following Ref. [67], we calculate the velocity distributions along the principal axes of the velocity dispersion tensor $\sigma_{i j}^{2}$. They are shown on Fig. 12. The Gaussian fit for each component and the Maxwellian fit for the velocity module are shown in red. A clear departure from these distributions is seen.

In order to model and parameterize these deviations, we introduce generalized Gaussian and Maxwellian distributions. The generalized Gaussian distribution with a mean velocity $\mu$, a velocity dispersion parameter $v_{0}$, and a Gaussianity parameter $\alpha$ is defined by

$$
\mathrm{f}(\vec{v})=\frac{1}{N\left(v_{0}, \alpha\right)} e^{-\left((v-\mu)^{2} / v_{0}^{2}\right)^{\alpha}}
$$

with a normalization factor $N\left(v_{0}, \alpha\right)=2 v_{0} \Gamma(1+1 / 2 \alpha)$. For $\alpha=1$, the usual Gaussian distribution is recovered. Similarly, the generalized Maxwellian distribution is defined by

$$
\mathrm{f}(\vec{v})=\frac{4 \pi v^{2}}{N\left(v_{0}, \alpha\right)} e^{-\left(v^{2} / v_{0}^{2}\right)^{\alpha}},
$$

with a normalization factor $N\left(v_{0}, \alpha\right)=4 \pi v_{0}^{3} \Gamma(1+3 / 2 \alpha) / 3$. For $\alpha=1$, we get the usual Maxwellian distribution.

For the velocity components, a fit with a generalized Gaussian distribution (in green in Fig. 12, panels a), b) and c)) shows that the velocity distribution given by the simulation is systematically more flat than a Gaussian distribution. This property can be described by the so-called Kurtosis parameter 
$K$, which compares the fourth-order moment with the square of the variance. A Kurtosis parameter $K<3$ corresponds to a distribution that is more flat than a Gaussian distribution with the same standard deviation, while $K>3$ corresponds to a distribution that is more peaked around the central value compared to the Gaussian one. The goodness-of-fit for each theoretical distribution is calculated using the Pearson's $\chi^{2}$ test. The values of $\chi^{2}$ (calculated with a number of bins $n_{b i n}=50$ ) printed in each panel of Fig. 12 show that the fit is considerably improved when using a generalized Gaussian distribution with $\alpha>1$ (the actual values of $v_{0}$ and $\alpha$ are estimated on the basis of the standard deviation $\sigma$ and the Kurtosis parameter $K$ extracted from the velocity distribution). We also notice that along the principal direction "3", the velocity dispersion and the deviation from the Gaussian distribution are smallest. It appears that this direction is actually very close to the galactic pole axis.

For the velocity module, a clear deviation from a Maxwellian distribution (in red) is apparent, with a sharper tail drop at high velocity, particularly crucial for the inelastic recoil scenario (see Sec. 3.5). The goodness-of-fit is again improved with a generalized Maxwellian distribution with $\alpha>1$. However, even the best-fit values $\left(v_{0}=301.6 \mathrm{~km} / \mathrm{s}\right.$ and $\left.\alpha=1.55\right)$ do not model the simulation distribution in a satisfactory way. Indeed, while the generalized Gaussian distribution enables to fit both the standard deviation and the Kurtosis parameter, only the standard deviation can be accommodated in the case of the generalized Maxwellian distribution. It appears that the N-body velocity distribution is "fatter" than the best-fit generalized Maxwellian, with a Kurtosis parameter $K=2.39$ vs. $K=2.71$ respectively. In panel d) of Fig. 12, we show two possible fits with a generalized Maxwellian distribution, corresponding to $\alpha=1.5$ and $\alpha=1.95$. The former gives a better global fit for the whole distribution and for the low velocity bins. The latter gives a better fit of the high velocity tail. These models will be used and discussed in the direct detection section (see Sec. 3.4 for the elastic scenario and Sec. 3.5 for the inelastic scenario).

In dark matter-only simulations, one has a $4 \pi$ uncertainty on the Sun position. This freedom is lifted, at least partially, in simulations with baryons where the galactic plane can be identified. To determine it, we select star particles with $3<R<10 \mathrm{kpc}$ to avoid the galactic bulge. We have $N_{\text {star }}=1,935,104$ in this selection. The galactic plane is then rotated onto the $x y$ plane by diagonalizing the position tensor. In this new reference frame, denoted by $\left\{\overrightarrow{1}_{x}, \overrightarrow{1}_{y}, \overrightarrow{1}_{z}\right\}$, we select DM particles with $7<R<9 \mathrm{kpc}$ and $|z|<1 \mathrm{kpc}$, corresponding to particles located in a ring around the galactic plane. The number of particles in this selection is $N_{\text {ring }}=2,662$. Fig. 13 shows the corresponding velocity distributions in cylindrical coordinates $\{r, \phi, z\}$. Anisotropy is manifest. There is a significant rotation along the galactic disk, with $\left\langle v_{\phi}\right\rangle=35.2 \mathrm{~km} / \mathrm{s}$. For the radial and $z$ velocity components, the mean of the distribution is compatible with zero. The velocity dispersions and Kurtosis parameters also exhibit some anisotropy in the $z$ direction compared to the galactic plane. Again, the distribution of the velocity module indicates a strong deviation from a Maxwellian distribution.

\subsection{Dark disk, halo rotation and local density}

As pointed out in Ref. [70], the rotation of the DM halo observed in Fig. 13 is expected in $\Lambda$ CDM cosmology with hierarchical structure formation. At relatively low redshift, $z<2$, merging satellite galaxies are preferentially dragged towards the already well-formed disk of a host galaxy, where they are disrupted by tidal forces. The accreted material settles in a thick stellar disk and a thick dark matter disk, dubbed the dark disk. The vertical distribution of the stars of the host galaxy thickens due to the impact, but all new stars form in a thin disc [82, 83, 84]. Observational evidence for a thick stellar disk in the Milky Way has been first pointed out by Gilmore and Reid (1983) [85]. Although the detailed morphology and some quantitative properties of the final galaxy depend on its merger history, the existence of a thick stellar disk and a co-rotating dark disk in the $\Lambda$ CDM paradigm seems quite robust. Using three cosmological hydrodynamics simulations, Read et al. [69] conclude that the accreted dark matter can contribute $\sim 0.25-1.5$ times the non-rotating halo density at the solar position. Moreover, the DM $v_{\phi}$ distribution is best-fit with a double Gaussian, one for the non-rotating halo component, and one for the rotating accreted DM. The rotation lag of the rotating component is in the range $0-150 \mathrm{~km} / \mathrm{s}$. A large value of the rotation lag, corresponding to a small halo circular speed, is only found for galaxies which had no significant merger after a redshift $z=2$. The importance of the dark disk for the prediction of DM direct detection rates has been acknowledged by several 
authors [71, 69]. It could lead to an increase by up to a factor of 3 in the $5-20 \mathrm{keV}$ recoil energy range. The signal modulation can also be boosted and the modulation phase is shifted. However, in Ref. [86], the authors use high-resolution simulations of accretion events in order to bracket the range of co-rotating accreted dark matter. They find that the Milky Way's merger history must have been unusually quiescent compared to median $\Lambda \mathrm{CDM}$ expectations. As a result, the fraction of accreted dark matter near the Sun is less than $20 \%$ of the host halo density, and does not change the likelihood of detection significantly.

To investigate this topic further, we select star particles with $7<R<9 \mathrm{kpc}$ and $|z|<3 \mathrm{kpc}$, we have $N_{\text {star }}=143,320$. The distribution of $v_{r}$ and $v_{\phi}$ are shown on Fig. 4 . We observe that the dark matter and the star particles are indeed co-rotating in the solar neighborhood. The mean tangential velocity is $\left\langle v_{\phi}\right\rangle=201 \mathrm{~km} / \mathrm{s}$ but tends towards $\left\langle v_{\phi}\right\rangle=225 \mathrm{~km} / \mathrm{s}$ for stars closer to the galactic plane, which is consistent with Milky Way rotation curve data [47]. Moreover, the $v_{\phi}$ distribution is clearly bimodal, a double Gaussian gives a reasonable fit. The low velocity component $(\mu=175 \mathrm{~km} / \mathrm{s})$ has a larger velocity dispersion parameter $\left(v_{0}=72 \mathrm{~km} / \mathrm{s}\right)$ than the high velocity component $(\mu=270 \mathrm{~km} / \mathrm{s}$, $v_{0}=30 \mathrm{~km} / \mathrm{s}$ ). To check whether these correspond to the thick and thin disks populations, we take subsets of the previous selected star particles with $0<v_{\phi}<150 \mathrm{~km} / \mathrm{s}$ and $v_{\phi}>275 \mathrm{~km} / \mathrm{s}$ respectively, and calculate the disc scale height as well as the total velocity dispersion. We obtain $z_{d}^{\text {thick }} \simeq 3 \mathrm{kpc}$, $z_{d}^{\text {thin }} \simeq 0.67 \mathrm{kpc}, \sigma_{\text {tot }}^{\text {thick }} \simeq 165.5 \mathrm{~km} / \mathrm{s}, \sigma_{\text {tot }}^{\text {thin }} \simeq 65.5 \mathrm{~km} / \mathrm{s}$. These values are higher than those measured for the Milky Way: from SDSS tomographic results, $z_{M W}^{\text {thick }} \simeq 1 \mathrm{kpc}, z_{M W}^{\text {thin }} \simeq 0.35 \mathrm{kpc}$ [87]; for the velocity dispersion, $\sigma_{M W}^{\text {thick }} \simeq 85 \mathrm{~km} / \mathrm{s}$ [88], $\sigma_{M W}^{\text {thin }} \simeq 40 \mathrm{~km} / \mathrm{s}$ [89]. Although the thick and thin stellar disks could be revealed by applying severe cuts on $v_{\phi}$, the inferred values of the disk scale height and the total velocity dispersion are not reliable, they probably overestimate the correct values by up to a factor 2. Hence, we stay inconclusive regarding the consistency of stellar populations in this simulation with Milky Way observations.

The existence of two DM components in the solar neighborhood cannot be assessed undoubtfully in this simulation due to the limited local resolution. Nevertheless, as shown on panel b of Fig. 13, a fit with a double Gaussian gives a better description of the $v_{\phi}$ distribution than with a single Gaussian. From this fit, it appears that the mean velocity of the rotating component is comparable for DM and for stars. The dark disk particles also have a smaller velocity dispersion. Moreover, it appears that the dark disk constitutes around $25 \%$ of the total local density in the solar neighborhood. The value of this fraction can be further checked by calculating average densities from the simulation. For DM particles in the spherical shell $7<R<9 \mathrm{kpc}$, we find $\left\langle\rho_{D M}\right\rangle=0.31 \mathrm{GeV} / \mathrm{cm}^{3}$, in agreement with what is found in DM only simulations and the commonly adopted value in this field, $\rho_{D M}=0.3 \mathrm{GeV} / \mathrm{cm}^{3}$. However, when we restrict DM particles to a ring of thickness $\delta z$ around the galactic disk, the average density is higher. We find $\left\langle\rho_{D M}\right\rangle=0.37 \mathrm{GeV} / \mathrm{cm}^{3}$ for $\delta z / 2=1 \mathrm{kpc}$ and $\left\langle\rho_{D M}\right\rangle \simeq 0.39 \mathrm{GeV} / \mathrm{cm}^{3}$ for $\delta z / 2 \leq 0.1 \mathrm{kpc}$. The density increase near the galactic disk is indeed about $25 \%$ of the total density in the solar neighborhood.

The extent of the density increase is shown on Fig. 5. On the left panel, DM particles with $7<R<9 \mathrm{kpc}$ are partitioned into slices of thickness $\delta z=2 \mathrm{kpc}$ parallel to the plane $x y$ (galactic plane), and the average density is shown as a function of $z$. If the halo was close to spherical, there would be no bump around $z=0$. On the right panel, we show the disk to shell density ratio for different planes. For the galactic plane, the density increase due to the dark disk is typically between 15 and $30 \%$ for $R \leq 25 \mathrm{kpc}$. For other planes, the ratio oscillates around 0.9 , except when satellite halos are encountered, this is the case for the plane $y z$ and $R \simeq 13 \mathrm{kpc}$, as illustrated by the density maps of Fig. 6. With the dark disk component, the entire DM halo appears with an oblate shape. The local density increase due to the dark disk is relatively mild in this simulation, compared to more extreme cases [69]. Therefore, following the results of Ref. [71], we do not expect a strong increase of the direct detection signal in this simulation compared to a standard Maxwellian halo.

Finally, we checked that the DM rotation is not limited to a thin galactic plane but is present in a rather thick dark disk. In particular, Fig. 7 shows the variation of the mean tangential velocity $v_{\phi}$ as a function of $R$ for $z=0$ (left panel), and as a function of $z$ for $r=8 \mathrm{kpc}$ (right), where $r=\sqrt{x^{2}+y^{2}}$ is the polar radius. A significant rotation is found in the entire galactic plane, up to $R=100 \mathrm{kpc}$. For $r=8 \mathrm{kpc}$, the rotation remains important up to heights of $\sim 15 \mathrm{kpc}$ from the galactic plane. 


\section{Direct detection experiments and signals}

Direct detection experiments aim to measure the energy deposited during scatterings of Weakly Interacting Massive Particles (WIMPs) with the detector material. Given the weak interaction rate and the low expected signal, radiopurity of the material and control of other backgrounds are essential although experimentally challenging. In order to attenuate these difficulties, one possibility is to look for a typical signature of the signal that is hard to mimic with a background noise. The DAMA/Libra experiment [41] is the only one that has observed a positive signal with such a signature, namely the annual modulation of the event rate. However, as the signal could not be confirmed by any other experiment, neither with a heavier or a lighter target, the compatibility of the DAMA result and the exclusion limits set by these other experiments becomes more and more controversial [90, 91].

Ways of reconciliation have been sought in the detector characteristics (quenching factors uncertainties and channelling effects [92, 93]), in particle physics uncertainties (nuclear form factors [94], nucleon effective couplings [95]) or scenarios (elastic scattering [96, 97, 98, 99, 100], inelastic scattering [101, 102, 103, 60, 104, 105]), and in the Dark Matter halo velocity distribution [106, 107, 43, 108, 44, 60].

In this section, we give all the relevant steps necessary to compute the spin independent scattering signal of Dark Matter on nuclei in direct detection experiments. Reference values for all the useful parameters and quantities will be given, and discussed in light of the DAMA controversy. In this paper, the accent will be put on the impact of a realistic velocity distribution (as given by our cosmological simulation with gas and stars) on the direct detection signal and its modulation. In particular, we will focus on how the fit to DAMA and the other exclusion limits change compared to a standard Maxwellian halo. Both the elastic and the inelastic cases will be covered. For the influence of other parameters, the reader will be referred to the existing literature.

\subsection{Event rate formalism}

We consider collisions between dark matter particles from the Galactic halo and the nuclei of a given low background detector. The relevant characteristics of the halo are the local density of dark matter, taken to have the fiducial value $\rho_{D M}=0.3 \mathrm{GeV} / \mathrm{cm}^{3}$ at the Sun's location, and the local distribution of velocities $\mathrm{f}(\vec{v})$ with respect to the Earth.

The differential event rate of nuclear recoils as a function of the recoil energy $E_{R}$ is conveniently factored as

$$
\frac{d \mathcal{R}}{d E_{R}}=\frac{\rho_{D M}}{M_{D M}} \frac{d \sigma}{d E_{R}} \eta\left(E_{R}, t\right)
$$

where $M_{D M}$ is the WIMP mass, $d \sigma / d E_{R}$ encodes all the particle and nuclear physics factors, and $\eta\left(E_{R}, t\right)$ is the mean inverse velocity of incoming DM particles that can deposit a recoil energy $E_{R}$. The time dependence of the velocity distribution is induced by the motion of the Earth around the Sun, which leads to a seasonal modulation of the event rate $[109,110]$.

The total recoil rate per unit detector mass in a given energy bin $\left[E_{1}, E_{2}\right]$ is obtained by integrating Eq. (3),

$$
\mathcal{R}(t)=\int_{E_{1}}^{E_{2}} d E_{R} \epsilon\left(E_{R}\right)\left(\frac{d \mathcal{R}}{d E_{R}} \star G\left(E_{R}, \sigma\left(E_{R}\right)\right)\right),
$$

where $\epsilon$ is the efficiency of the detector and the finite energy resolution of the experiment is taken into account by convoluting the differential rate with a Gaussian distribution with spread $\sigma\left(E_{R}\right)$. For detectors made of several elements, the total rate is the average of the rates $\mathcal{R}_{i}(t)$ for each component $i$, weighted by its mass fraction $f_{i}$

$$
\mathcal{R}(t)=\sum_{i} f_{i} \mathcal{R}_{i}(t)
$$

Finally the expected number of observed events per unit time is the product of the total rate times the detector mass $M_{\text {det }}$.

The particle physics is enclosed in the term $d \sigma / d E_{R}$, which is generally parameterized as

$$
\frac{d \sigma}{d E_{R}}=\frac{M_{N} \sigma_{n}^{0}}{2 \mu_{n}^{2}} \frac{\left(f_{p}^{2} Z+(A-Z) f_{n}^{2}\right)^{2}}{f_{n}^{2}} F^{2}\left(E_{R}\right)
$$


where $M_{N}$ is the nucleus mass, $\mu_{n}$ is the reduced WIMP/neutron mass, $\sigma_{n}^{0}$ is the zero momentum WIMP-neutron effective cross-section, $Z$ and $A$ are respectively the number of protons and the atomic number of the element, $f_{p}\left(f_{n}\right)$ are the WIMP effective coherent coupling to the proton (resp. neutron), and $F^{2}\left(E_{R}\right)$ is the nuclear form factor. In the sequel, we will consider the case of scalar interactions, most relevant for the elastic scattering scenario, for which $f_{p} \simeq f_{n}$ and the case of weak vector interactions through a $Z$ boson, most relevant for the inelastic scattering scenario, for which $f_{p}=4 \sin ^{2} \theta_{W}-1$ and $f_{n}=1$. The form factor $F^{2}\left(E_{R}\right)$ characterizes the loss of coherence for non zero momentum transfer. We use the simple parameterization given by Helm [111, 94], defined as

$$
F\left(E_{R}\right)=3 e^{-q^{2} s^{2} / 2} \frac{J_{1}(q r)}{q r},
$$

with $q=\sqrt{2 M_{N} E_{R}}, s=1.0 \mathrm{fm}, r=\left\{\left(1.23\left(m_{N} / m_{n}\right)^{1 / 3}-0.6\right)^{2}+0.63 \pi^{2}-5 s^{2}\right\}^{1 / 2}$ fm the effective nuclear radius, $J_{1}$ is a spherical Bessel function of the first kind. This form factor is optimal for scattering on Iodine [94]. For simplicity we use the same form factor for all targets (more accurate form factor are off by at most $\mathcal{O}(20 \%)$, for some targets and at large recoil energies [112]).

Finally, the velocity distribution appears in the quantity

$$
\eta\left(E_{R}, t\right)=\int_{v_{m i n}} d^{3} \vec{v} \frac{\mathrm{f}(\vec{v}(t))}{v},
$$

where $\vec{v}$ the WIMP velocity wrt the Earth and $v_{\text {min }}$ is the minimum velocity needed to provoke a recoil inside the detector. Two cases need to be considered. In the elastic scattering scenario, a dark matter particle is simply scattered off a nucleus. In the inelastic scattering scenario, a dark matter particle $D M_{1}$ is supposed to scatter into a slightly heavier state $D M_{2}$, with a mass splitting $\delta=M_{D M_{2}}-M_{D M_{1}} \sim 100$ $\mathrm{keV}$. In this scenario, which has been first proposed in Ref. [101] and confronted to the recent data in Refs. [102, 103, 60, 104, 105], a much broader range of dark matter candidates may both fit DAMA and be consistent with the other experiments. The threshold velocity is given by

$$
v_{\text {min }}=\sqrt{\frac{1}{2 M_{N} E_{R}}}\left(\frac{M_{N} E_{R}}{\mu}+\delta\right),
$$

with $\mu$ the WIMP-nucleus reduced mass. This formula encompasses both the elastic $(\delta=0)$ and the inelastic $(\delta \neq 0)$ scattering scenarios.

When the velocity distribution in the galactic frame $\mathrm{f}_{\text {gal }}(v)$ is isotropic, $\eta$ is given by

$$
\eta=\frac{2 \pi}{v_{\oplus}} \int_{v_{-}}^{v_{+}}\left(\mathrm{F}\left(v_{e s c}\right)-\mathrm{F}(v)\right) d v
$$

with $v_{ \pm}=\min \left\{v_{e s c}, v_{\min } \pm v_{\oplus}\right\}, v_{e s c}$ is the galactic escape velocity, $\vec{v}_{\oplus}(t)$ is the Earth's velocity in the galactic frame, which varies with the time of year $t$, and $\mathrm{F}(v)=\int v \mathrm{f}_{\text {gal }}(v) d v$. In deriving this formula, we have used the realistic assumption $v_{e s c}>v_{\oplus}$ at any time $t$. Notice that $\mathrm{F}(v)$ is an even function of $v$, because $\mathrm{f}_{\text {gal }}$ only depends on the modulus $|\vec{v}|$ of the velocity. For a standard Maxwellian distribution with a mean velocity $v_{0}$,

$$
\mathrm{f}_{\text {gal }}(\vec{v})=\frac{1}{N\left(v_{e s c}\right) \pi^{3 / 2} v_{0}^{3}} e^{-v^{2} / v_{0}^{2}} \quad \text { for } v<v_{e s c},
$$

where $N\left(v_{\text {esc }}\right)$ is a normalization factor, Eq. (10) reads

$$
\eta=\frac{1}{2 N\left(v_{e s c}\right) v_{\oplus}}\left\{\operatorname{Erf}\left(\frac{v_{+}}{v_{0}}\right)-\operatorname{Erf}\left(\frac{v_{-}}{v_{0}}\right)-\frac{2\left(v_{+}-v_{-}\right)}{\sqrt{\pi} v_{0}} e^{-v_{e s c}^{2} / v_{0}^{2}}\right\},
$$

which agrees with the result of Ref. [113]. It might seem curious that Eq. (10) is expressed as a definite integral between $v_{-}$and $v_{+}$while particles with any velocity above $v_{\text {min }}$ should contribute to $\eta$. However, the function $F(v)$ is itself an integral on the velocity distribution. Also the dependence in $v_{e s c}$ is now explicit. In the rest of this paper, we will assume an escape velocity $v_{e s c}=600 \mathrm{~km} / \mathrm{s}$, 
somewhat on the upper part of the expected range, $498 \mathrm{~km} / \mathrm{s}<v_{e s c}<608 \mathrm{~km} / \mathrm{s}$ [108]. Modifications of the velocity distributions and their impact on the fits, have been discussed in various works, see for instance Refs. [43, 44, 60, 104].

The seasonal modulation of the event rate is caused by the variation of the Earth's velocity throughout the year. Following Refs. [106, 55], in the galactic reference frame such that $\overrightarrow{1}_{X}$ is pointing towards the galactic center, $\overrightarrow{1}_{Y}$ is along the disk rotation, and $\overrightarrow{1}_{Z}$ is pointing towards the galactic north pole, we write

$$
\begin{aligned}
\vec{v}_{\oplus}(t) & =\vec{v}_{\odot}+\vec{v}_{\mathrm{EO}}(t) \\
\vec{v}_{\odot} & =\vec{v}_{c}+\vec{v}_{\odot, p e c}=(0,220,0)+(10,5,7) \mathrm{km} / \mathrm{s} \\
\vec{v}_{\mathrm{EO}}(t) & =v_{\mathrm{EO}}\left(\vec{e}_{1} \sin \omega\left(t-t_{1}\right)-\vec{e}_{2} \cos \omega\left(t-t_{1}\right)\right)
\end{aligned}
$$

where the velocity $\vec{v}_{\odot}$ of the Sun with respect to the halo [114] is decomposed as the sum of the galactic disk circular velocity $\vec{v}_{c}$ and a peculiar velocity $\vec{v}_{\odot, p e c}$, the Earth mean orbital velocity is $v_{\mathrm{EO}}=29.8 \mathrm{~km} / \mathrm{s}$, with $\omega=2 \pi / T$ and a period $T=1$ year, $t_{1}=79.62$ days is the time of the vernal equinox, and $\vec{e}_{1}=(-0.067,0.4927,-0.8676), \vec{e}_{2}=(-0.9931,-0.1170,0.01032)$ (the Earth's orbital eccentricity is neglected). Therefore the Earth velocity follows a cosine function. As $v_{\mathrm{EO}} \ll v_{\odot}$, we have

$$
v_{\oplus}(t) \simeq v_{\odot}+v_{\mathrm{EO}} \sin \gamma \cos \omega\left(t-t_{0}\right)
$$

with $\gamma \simeq 29.3^{\circ}$ is the angle between the ecliptic plane perpendicular axis and $\vec{v}_{\odot}$. The Earth velocity is maximal for $t=t_{0}$, with

$$
t_{0}=t_{1}+\frac{\pi}{2 \omega}+\frac{1}{\omega} \arctan \frac{\vec{v}_{\odot} \cdot \vec{e}_{2}}{\vec{v}_{\odot} \cdot \vec{e}_{1}} \simeq 151.5 \text { days } .
$$

With these values, the Earth velocity modulation amplitude amounts to $6.2 \%$. As pointed out in Ref. [115], the circular velocity of the Sun may be larger than the value $v_{c}=220 \mathrm{~km} / \mathrm{s}$ usually considered. Also, if the DM halo is co-rotating with the galactic disk, the relative velocity relevant for calculating the direct detection signal is effectively reduced. In Fig. 8, the circular velocity is varied between $100 \mathrm{~km} / \mathrm{s}$ and $300 \mathrm{~km} / \mathrm{s}$, the corresponding phase $t_{0}$ increases with $v_{c}$, from $t_{0}=144$ to $t_{0}=153$ days.

For an isotropic velocity distribution, the differential rate also follows a temporal cosine function at first order in $v_{\mathrm{EO}} / v_{\odot}$. Indeed, the only time dependent factor in Eq. (3) is $\eta$, for which we get, in the limit $v_{e s c}>v_{+}$

$$
\eta\left(E_{R}, t\right) \simeq \eta_{0}\left(E_{R}\right)+\eta_{1}\left(E_{R}\right) \frac{v_{\mathrm{EO}}}{v_{\odot}} \sin \gamma \cos \omega\left(t-t_{0}\right)
$$

with

$$
\begin{aligned}
\eta_{0}\left(E_{R}\right) & =\eta\left(E_{R}, v_{\mathrm{EO}}=0\right) \\
\eta_{1}\left(E_{R}\right) & =\frac{2 \pi}{v_{\odot}} \int_{v_{-}^{0}}^{v_{+}^{0}} F(v) d v-2 \pi\left(F\left(v_{+}^{0}\right)+F\left(v_{-}^{0}\right)\right)
\end{aligned}
$$

with $v_{ \pm}^{0}=v_{\min }\left(E_{R}\right) \pm v_{\odot}$. It should be stressed that the time of year $t=t_{0}$ does not necessarily correspond to a maximum of the event rate. For low energy events, Eq. (17) shows that it is a minimum instead, as $\eta_{1}$ becomes negative.

In this paper, we also need to consider discrete velocity distributions as the output of a simulation gives a list of particles with their position and velocity. If we denote by $N_{\text {part }}$ the number of particles, and by $\vec{v}_{i}$ the velocity of the particle $i$ in the galactic frame, the distribution can be written as

$$
\mathrm{f}_{\text {gal }}(\vec{v})=\frac{1}{N_{\text {part }}} \sum_{i} \delta\left(\vec{v}-\vec{v}_{i}\right)
$$

where $\delta$ is the Dirac delta distribution in 3 dimensions. Therefore, for $\eta$, we get in this case

$$
\eta=\frac{1}{N_{\text {part }}} \sum_{i} \frac{1}{w_{i}} H\left(w_{i}-v_{\min }\right)
$$


with $w_{i}=\left|\vec{v}_{i}-\vec{v}_{\oplus}\right|$ and $H(x)$ is the Heaviside step function. To compute the total rate, or the modulation amplitude in some energy bin, we need to integrate the differential event rate. For discrete distributions, we take advantage of the step-like shape of the differential event rate for a given flow $\vec{v}_{i}$ to get accurate results.

\subsection{DAMA modulation data}

The former DAMA/NaI [36] and its follow-up DAMA/LIBRA [41] are experiments run at the LNGS at Gran Sasso, Italy using $\mathrm{NaI}(\mathrm{Tl})$ crystals as target. They are designed to detect the dark matter recoil off nuclei through the model independent annual modulation signature which is due to the motion of the Earth around the Sun. The experimental results obtained by DAMA/LIBRA, with an exposure of 0.53 ton $\times$ yr collected over 4 annual cycles, combined with the ones of DAMA/NaI, for an exposure of 0.29 ton $\times$ yr collected over 7 annual cycles, corresponding to a total exposure of 0.82 ton $\times$ yr, show a modulated signal with a confidence level of $8.2 \sigma$ [41].

The DAMA modulation data, reproduced on Fig. 9, are obtained by adjusting (with a likelihood function) the observed event rate in each energy bin $k$ to a temporal cosine function

$$
S_{k}=S_{0, k}+S_{m, k} \cos \omega\left(t-t_{0}\right),
$$

where $S_{0, k}$ is the constant part of the signal, $S_{m, k}$ is the modulation amplitude. The DAMA collaboration uses the value $t_{0}=152.5$ days (2nd of June) for the phase corresponding to a maximal Earth velocity and a maximal event rate. It should be stressed that the true value of $t_{0}$ depends in general upon the velocity of the Sun, and upon the DM velocity distribution. However, for an isotropic distribution, Eqs. (15-16) show that the phase $t_{0}$ is completely determined once the velocity of the Sun in the galaxy is specified. For the low energy region where the signal modulation is present, the experimental value of $t_{0}$ has been determined by the DAMA collaboration. When the phase is allowed to vary freely, and fitting again the event rate in the energy interval $2 \leq E e e \leq 6 \mathrm{keV}$ with a cosine function, the best-fit value for $t_{0}$ is

$$
t_{0}=144.0 \pm 7.5 \text { days }(1 \sigma)
$$

The DAMA spectrum is given in keVee (electron-equivalent keV). The observed energy released in scintillation light is related to the nucleus recoil energy through a so-called quenching factor $q$, $E_{\text {scint. }}=q \cdot E_{\text {recoil }}$. This expresses the fact that a recoiling nucleus may loose energy by collisions with other nuclei, hence in the form of heat, or through collisions with electrons, which create scintillation light. The reference values for Iodine and Sodium are respectively $q_{I}=0.09$ and $q_{N a}=0.3$. However it has been pointed that the so-called channelled events may play a role [92, 93]. This refers to events in which a recoiled nucleus moves along the axis of the NaI crystal, losing most of its energy by collisions with electrons, in which case the quenching factor may be larger, up to $q \approx 1$. Once channelling is taken into account, collisions of light WIMPs with Iodine become relevant, while recoils on Sodium become negligible in all scenarios $[93,116,42,113,43,117]$. We use the fraction $f$ of channelled events given in Ref. [43],

$$
f_{\mathrm{Na}}\left(E_{R}\right)=\frac{e^{-E_{R} / 18}}{1+0.75 E_{R}}, \quad f_{\mathrm{I}}=\frac{e^{-E_{R} / 40}}{1+0.65 E_{R}},
$$

where the recoil energy $E_{R}$ is in $\mathrm{keV}$. We have verified that the other parameterizations found in the literature $[103,42,113]$ give identical results. Finally, we use the energy resolution of the detector given by the collaboration [41]

$$
\frac{\sigma(E)}{E}=\frac{0.45}{\sqrt{E}}+0.0091
$$

and a detector efficiency $\epsilon=1[41,113]$.

To fit the observed energy spectrum of Fig. 9 with a theoretical model, we use a goodness-of-fit (GOF) method with a $\chi^{2}$ metric, for the 12 first bins of data, with width 0.5 keVee, from 2.0 to 8.0 keVee [41].

$$
\chi^{2}=\sum_{i=1}^{n} \frac{\left(S_{i}-S_{i}^{o b s}\right)^{2}}{\sigma_{i}^{2}}
$$


where $S_{i}$ is the predicted value of the modulation amplitude in the bin number $i, S_{i}^{\text {obs }}$ is the value reported by DAMA and $\sigma_{i}$ is the corresponding experimental uncertainty. As data above 8.0 keVee are essentially compatible with no modulation signal, including them in the $\chi^{2}$ fit enlarges the allowed regions in the parameter space. For explicit numerical values of $S_{i}^{\text {obs }}$, we refer the reader to Table III of Ref. [113]. As emphasized earlier, these values were obtained by the DAMA collaboration with the hypothesis $t_{0}=152.5$. To be consistent with their analysis and to facilitate comparisons with previous analyses, we will also use this fixed value of $t_{0}$ for the determination of the allowed regions in the parameter space, although the true phase corresponding to the maximal event rate might be different for some energy bins. For two parameters $\left(M_{D M}\right.$ and $\sigma$ in the elastic scenario, and $\delta$ and $M_{D M}$ in the inelastic scenario), the $\chi^{2}$ metric has 10 degrees of freedom, therefore the $90 \%$ (99\% and $99.9 \%$ ) confidence level (CL) corresponds to $\chi^{2}<16.0$ (resp. $\chi^{2}<23.2$ and $\chi^{2}<29.6$ ).

\subsection{Other experiments exclusion limits}

So far all the other direct detection experiments searching for dark matter are compatible with null results. In this section we briefly describe the experiments that lead to the most constraining limits on both the elastic and the inelastic scenarios.

In the elastic scenario, light nuclei, like Aluminum $(A=27)$ and Silicon $(A=29)$, are more sensitive to light WIMPs scattering $M_{D M} \sim$ multi-GeV, and provide the strongest upper bounds on the allowed parameter space favored by DAMA. In the inelastic scenario, which involves heavier candidates, experiments made of heavy nuclei, like Iodine $(A=127)$, Xenon $(A=129)$ and Tungsten $(A=184)$ are the most constraining ones. Germanium $(A=73)$ made detectors fall in between.

In computing the rate of Eq. (4) for each experiment, we uniformly assume that the small number of events seen, if any, are signals from dark matter and we use the Poisson statistics to find the parameter space excluded at a given confidence level (CL). Integrating over the exposure time, the upper bounds are obtained by requiring that the total number of events $N_{t o t}$ is compatible with the number of observed events at $99.9 \%$ of CL, while Ref. [104] uses 99\% CL. This means that we take less severe constraints, leading to slightly smaller excluded regions. We have checked that the exclusion curves that we obtain reproduce fairly well the published $90 \%$ CL upper bounds for each experiment. In the following we describe the main features of the experiments considered, their particular characteristics, and the $\chi^{2}$ function adopted.

CDMS: The Cryogenic CDMS experiment at Soudan Underground Laboratory operates Ge and Si made solid-state detectors. For a heavy WIMP, the Ge data are more constraining: we have considered the ensemble of the three released runs, with respectively an exposure of $19.4 \mathrm{~kg}$-day [118], $34 \mathrm{~kg}$ day [119] after cuts and a total exposure of $397.8 \mathrm{~kg}$-days before cuts for the third run [37]. The sensitivity to nuclear recoils is in the energy range between $10-100 \mathrm{keV}$ while the efficiencies and the energy resolution of the detectors are given in [97]. For the third run, the efficiency is parameterized as $\epsilon\left(E_{R}\right)=0.25+0.05\left(E_{R}-10\right) / 5$ for $10 \mathrm{keV}<\mathrm{E}_{\mathrm{R}}<15 \mathrm{keV}$ and $\epsilon\left(E_{R}\right)=0.30$ for $E_{R} \geq 15 \mathrm{keV}$. The searches on Si are more sensitive to light DM candidates. For the run released in Ref. [120], two $100 \mathrm{~g}$ Si ZIP detectors were used, and the data set corresponds to 65.8 live days. The energydependent efficiency has been modeled in Ref. [113] as $\epsilon\left(E_{R}\right)=0.80 \times 0.95\left(0.10+0.30\left(E_{R}-5\right) / 15\right)$ for $5 \mathrm{keV}<\mathrm{E}_{\mathrm{R}}<20 \mathrm{keV}$ and $\epsilon\left(E_{R}\right)=0.80 \times 0.95\left(0.40+0.10\left(E_{R}-20\right) / 80\right)$ for $20 \mathrm{keV}<\mathrm{E}_{\mathrm{R}}<100 \mathrm{keV}$. Two candidate events with energies $E_{R} \simeq 55 \mathrm{keV}$ and $E_{R} \simeq 95 \mathrm{keV}$ were observed, consistent with zero event once expected neutron background is taken into account. For the run released in Ref. [119], the total exposure is $12 \mathrm{~kg}$-day after cuts, and the energy range has a higher minimum threshold $7 \mathrm{keV}<\mathrm{E}_{\mathrm{R}}<100 \mathrm{keV}$.

For zero observed event and Poisson distributed data, the $\chi^{2}$ function reduces to

$$
\chi^{2}=2 N_{t o t}
$$

where $N_{\text {tot }}$ is the total predicted number of events in the entire range of energy, for given values $\left(p_{1}, p_{2}, \ldots\right)$ of parameters $\left(P_{1}, P_{2}, \ldots\right)$ of the tested model. Therefore, for a $99.9 \%$ CL limit and two parameters, the exclusion limit is obtained by requiring that $N_{\text {tot }}$ is less than 6.9 . 
XENON10: XENON10 is a dual-phase Xenon chamber operating at LNGS, with a total exposure of $316.4 \mathrm{~kg}$-days and 10 candidate events in the recoil energy range between $4.5-26.9 \mathrm{keV}[38,39]$. For the data analysis, we follow Ref. [43], using the 7 energy bins provided by the collaboration and a $\chi^{2}$ for Poisson distributed data

$$
\chi^{2}=2 \sum_{i}\left\{N_{i}^{\text {pred }}+B_{i}-D_{i}+D_{i} \log \left(\frac{D_{i}}{N_{i}^{\text {pred }}+B_{i}}\right)\right\},
$$

with $N_{i}^{\text {pred }}$ is the predicted number of events in the $i$ th bin, $B_{i}=(0.2,0.3,0.2,0.8,1.4,1.4,2.7)$ is the expected background, $D_{i}=(1,0,0,0,3,2,4)$ is the number of detected events, and the log term is zero if $D_{i}=0$. For a $99.9 \%$ CL limit and two parameters, the value of the $\chi^{2}$ function must be less than 13.8. As observed in Ref. [43], using a constant nuclear recoil scintillation efficiency $\mathcal{L}_{\text {eff }}=0.19$ gives a slightly underestimated energy threshold of $4.5 \mathrm{keV}$. Correcting for this bias leads to a slightly less severe exclusion limit. In this paper however, we will keep the energy bins as given by the XENON collaboration. Finally, we can notice that this experiment is also sensitive to inelastic dark matter in a similar way as the DAMA experiment, due to the close proximity of the target nucleus masses.

CRESST: The CRESST-I and CRESST-II experiments at LNGS are mostly sensitive to light and heavy WIMPs respectively. In the first data release (CRESST-I) [121], the prototype detector module uses $\mathrm{Al}_{2} \mathrm{O}_{3}$ crystals as target, with a total exposure of $1.51 \mathrm{~kg}$-days covering the energy range between $0.6-20 \mathrm{keV}$. The energy resolution is given by $\sigma(E)=\sqrt{(0.519 \mathrm{keV})^{2}+(0.0408 E)^{2}}$ and the collaboration reports eleven observed events after cuts. Therefore, for Poisson distributed data, the number of predicted events cannot exceed 23 at $99.9 \%$ CL. For the second commissioned run of 2007 (CRESST-II) [122, 40], the target is changed to $\mathrm{CaWO}_{4}$. The presence of heavy nuclei of Tungsten enhances the sensitivity to spin-independent inelastic scatterings. The energy range for the CRESSTII Zora and Verena detectors is $12-100 \mathrm{keV}$, with a total exposure of $47.9 \mathrm{~kg}$-days before cuts and an acceptance of 0.9 on Tungsten recoil. The collaboration measured seven events, leading to a limit $N_{\text {tot }}<16$ at $99.9 \%$ CL.

Other experiments are potentially relevant, like ZEPLIN, CoGeNT and TEXONO. Also, the total rate observed by DAMA provides a constraint that excludes part of the parameter space. However, to avoid the cluttering of the figures, we do not include these limits in this paper, as they are weaker than the constraints presented.

\subsection{Results for the Elastic Scenario}

In the case of spin-independent elastic scatterings, the effective coherent couplings $f_{p}$ and $f_{n}$ of the WIMP to the proton and the neutron have similar values. Therefore, the only relevant parameters are the WIMP mass $M_{D M}$ and the spin independent cross section on nucleon, here simply denoted as $\sigma$ (see Eq. (6)). The main result for the elastic scenario is shown on the four panels of Fig. 14, where the regions in the plane $M_{D M}-\sigma$ favored by DAMA are plotted against the exclusion limits set by CDMS and XENON10 (the excluded regions are on the right and above the curves). From top left, the predictions for different halos: a) standard Maxwellian, b) this simulation, c) generalized Maxwellian with $\alpha=1.5$, d) generalized Maxwellian with $\alpha=1.95$.

In the elastic scenario, two regions are compatible with DAMA. For the region on the right, which is totally excluded by the other experiments, the signal is due to quenched scatterings events on Iodine. The region on the left, due to channeled events on Iodine, is not totally excluded by CDMS. The recent results of XENON10 set however a stronger limit, so that the elastic solution becomes very marginal. Here we have chosen to fit the DAMA data on 12 bins, which yields a smaller allowed region than with 36 bins. For a standard Maxwellian halo, with $v_{0}=220 \mathrm{~km} / \mathrm{s}$, it appears that the $99.9 \%$ CL region of DAMA is totally excluded by XENON10 at $99.9 \%$ CL.

For the simulation halo, the direct detection prediction is made by analyzing the phase-space of the central and best resolved Milky-Way sized galactic DM halo. The local structure is obtained by selecting the particles $\left(N_{\text {ring }}=2,662\right)$ located in a ring $(7 \leq R \leq 9 \mathrm{kpc}$ and $|z| \leq 1 \mathrm{kpc})$ around $R_{0}=8 \mathrm{kpc}$ in the galactic plane. Despite the small number of flows in this selection, which causes 
some numerical noise and artifacts in the $\chi^{2}$ plots, a few observations can be made. The DAMA regions are slightly smaller than in the Maxwellian case, and the best-fit points move to the left. Also, the region excluded by CDMS-Si slightly enlarges towards smaller cross sections. Moreover, the channeling region extends outside the XENON exclusion limit, so that the compatibility between DAMA and the other experiments is improved with the realistic halo from the simulation. While the best-fit point of the channeling region is still excluded, the $99.9 \%$ and $99 \%$ CL regions are not totally excluded anymore.

Several factors contribute to this improvement. The departure from a strict Maxwellian distribution has some impact, although moderate, as shown in panels $\mathrm{c}$ and d of Fig. 14. We took the two values $\alpha=1.5$ and $\alpha=1.95$ corresponding to the distributions shown in Fig. 12. As in the simulation, a displacement of the best-fit points towards smaller masses compared to the standard Maxwellian case is noticeable. On the contrary, if we take a Maxwellian halo with parameters tuned to fit as best as possible the simulation halo $\left(\rho_{D M}=0.37, v_{0}=239 \mathrm{~km} / \mathrm{s}\right.$, and smaller circular velocity $v_{c}=190 \mathrm{~km} / \mathrm{s}$ to reproduce the global average halo rotation velocity), the $99.9 \%$ CL DAMA region is still totally excluded by XENON. Other possible factors include the velocity dispersion anisotropy, shown on Fig. 13, and a general anisotropy in the velocity field, induced by the co-rotating DM component, which has a small lag velocity $v_{l a g} \simeq 75 \mathrm{~km} / \mathrm{s}$ compared to the galactic disk. A detailed modeling and discussion of the impact of these features will be presented in a subsequent paper [123].

The panels of Fig. 10 show the differential rate modulation for $2 \leq E e e \leq 6 \mathrm{keV}$ and the time dependence of the total signal in this energy range for the two best-fit points of Fig. 14. Large fluctuations are seen in the modulation amplitude as a function of energy, they can be interpreted as numerical noise due to the relatively small number of flows used to describe the local structure of the simulation halo around the Sun. On the contrary, the integrated rate between 2 and $6 \mathrm{keV}$ is a smooth, cosine-like function of time, with a peak around $t \simeq 150$ as expected. The number of flows is however too small to reliably indicate any deviation from $t_{0}=152.5$.

\subsection{Results for the Inelastic Scenario}

The dominance of inelastic interactions over elastic ones is achieved in a natural way when the scattering is mediated by a massive gauge boson [104]. In the simplest scenario, the mediator is the weak $S U(2)_{L}$ boson $Z$, so that no new gauge boson needs to be introduced beyond the Standard Model. The free parameters are therefore the mass $M_{D M}$ of the DM candidate, and its mass splitting $\delta$ with the nextto-lightest DM state. In this paper, we will only consider this situation. More involved models, where the cross section differs from the weak cross section $\sigma_{Z}$ can be found in Ref. [104].

Results for the inelastic scenario are summarized on Fig. 15. The DAMA favored regions are shown together with the exclusion limits from CDMS and CRESST-II (the excluded region extends on the left of each curve). As in the elastic scenario, the four panels are the predictions for different halos: a) standard Maxwellian, b) this simulation, c) generalized Maxwellian with $\alpha=1.5$, d) generalized Maxwellian with $\alpha=1.95$.

In the inelastic scenario, for a standard Maxwellian halo $\left(\rho_{D M}=0.3 \mathrm{GeV} / \mathrm{cm}^{3}, v_{0}=220 \mathrm{~km} / \mathrm{s}\right.$, $v_{e s c}=600 \mathrm{~km} / \mathrm{s}$ ), there are three $99 \%$ CL regions compatible with DAMA, which are all mainly due to quenched scatterings on Iodine. The region with $\delta \simeq 30 \mathrm{keV}$ is excluded by the constraints from both CDMS and CRESST. It is actually excluded by DAMA itself, as it leads to a year averaged rate higher than observed. The region with $\delta>50 \mathrm{keV}$ and $M_{D M}>1 \mathrm{TeV}$ is the largest in terms of parameter space. For a standard Maxwellian halo, this region is only marginally compatible with the exclusion limits of CDMS and CRESST. Let us notice that in a minimal framework with only the two DM states, and their coupling to the $Z$, the standard out-of-equilibrium freeze-out picture leads to a DM relic abundance higher than the value measured by WMAP [124], because the (co)annihilation rates are suppressed for $M_{D M} \gg M_{Z}$. For scalar candidates, the adjustable mass of the charged $S U(2)$ partner of the DM candidate enables to obtain the correct relic density for a whole range of mass at the $\mathrm{TeV}$ scale [125]. Finally, the DAMA region with $M_{D M}<100 \mathrm{GeV}$ is not excluded by other experiments. For these candidates, due to the proximity of the $Z$ pole, the relic abundance is lower than needed for WMAP unless some asymmetry in the dark sector prevents DM density from collapsing during the freeze-out [45].

The impact of the numerical noise from the finite number of flows in the simulation becomes more 
severe in the case of the inelastic scenario. Indeed, for inelastic interactions, only particles with a velocity high enough can produce a recoil. From Eq. (9), it follows that the minimum velocity $v_{*}$ corresponding to the energy $E_{*}=\mu \delta / M_{N}$ is given by $v_{*}=\sqrt{2 \delta / \mu}$. As a consequence, in the inelastic case, the signal is due to the high velocity tail of the distribution. As panel b of Fig. 15 shows, the DAMA region with $\delta \simeq 30 \mathrm{keV}$ is strongly affected by numerical noise. In this region, only a small number of flows with a velocity around $v_{*} \simeq 235 \mathrm{~km} / \mathrm{s}$ contribute to the time dependent modulation. The region with $M_{D M}<100 \mathrm{GeV}$ is also reduced, as it requires flows with a velocity higher than $v_{*} \simeq 700 \mathrm{~km} / \mathrm{s}$. The third region is probably the most reliable. Its deformed shape, compared to a Maxwellian halo, as well as its relative position to the exclusion curves are features that show that the velocity distribution of the simulation halo is not Maxwellian and not isotropic. In particular, the compatibility between DAMA and the other experiments is strongly improved. DAMA solutions at 90\% CL are found, while only solutions at 99\% CL were available in the case of the standard Maxwellian halo. This improvement of the compatibility can also be attested by the different position of the best-fit point for each case.

Results for a generalized Maxwellian halo are shown on panels $\mathrm{c}$ and $\mathrm{d}$ of Fig. 15. When $\alpha$ is increased, the DAMA regions shrink. For $\alpha=1.95$, the region with $\delta \simeq 30 \mathrm{keV}$ has disappeared. Most importantly, the improvement of the fit between DAMA and the other experiments for the largest region can be reproduced. For this region, the relative position of the best-fit point and the exclusion curves of CDMS and CRESST-II is better described by the distribution with $\alpha=1.95$, as this value fits better the high velocity tail of the velocity module distribution found in this simulation (see Fig. 12).

As in the elastic case, the differential rate modulation for $2 \leq E e e \leq 6 \mathrm{keV}$ and the time dependence of the total signal in this energy range for the three best-fit points of Fig. 15 are shown on Fig. 11. The impact of numerical noise is clearly seen in the time modulation, which can strongly differ from a cosine-like behavior.

\section{$4 \quad$ Summary \& Perspectives}

In this paper, we have analyzed the phase-space structure of a galactic halo extracted from an advanced cosmological N-body simulation which contains stars, gas, and DM, and used this information to make direct detection predictions.

Usually, such predictions are done with simplified astrophysical assumptions. The local phase-space structure in the solar neighborhood is taken as a smooth, isotropic Maxwellian distribution. However, it is known for quite some time that deviations should be expected in any realistic DM halo. The action of long-range gravitational forces, as well as the presence of a non-virialized halo component in the form of cold streams from the continuous secondary galactic infall [106, 107] already contribute to distort the simple picture of an isotropic Maxwellian halo. Cosmological numerical simulations done in the $\Lambda \mathrm{CDM}$ paradigm have demonstrated the hierarchical character of structure formation, leading to galactic structures with numerous sub-halos [126, 127]. Recent N-body simulations with very refined resolution, such as Aquarius [66] or Via Lactea [65] have even shown that non-Gaussianity and anisotropy (the velocities are rather in the radial direction) are present [43,60]. However, these simulations contain only DM particles, and cannot therefore provide a realistic description of a galaxy in a satisfactory way, despite the large number of particles. Dynamical interactions between the baryonic and the dark components of a galaxy are indeed expected to cause non negligible distortions on the phase-space structure of both components [81]. Recently, simulations with baryons have shown that the presence of a galactic disk drags merging satellites in a preferential direction [69], which leads to the formation of a thick stellar disc and a so-called dark disk.

In this simulation, it appears that the DM halo contains a dark disk component that is corotating with the galactic disk. The local DM density in the solar neighborhood has a value $\rho_{D M}=$ 0.37 to $0.39 \mathrm{GeV} / \mathrm{cm}^{3}$, consistent with the recent determination of Ref. [49], but higher than the usually quoted value of $0.3 \mathrm{GeV} / \mathrm{cm}^{3}$. Despite large fluctuations, the average circular velocity differs significantly from zero throughout a thick region of the halo. At the Sun's location, a double Gaussian provides a reasonable fit of the distribution of the tangential velocity $v_{\phi}$. We infer that the rotating DM component has a mean lag velocity $v_{l a g} \simeq 75 \mathrm{~km} / \mathrm{s}$ compared to the stellar disc, and contributes 
to around $25 \%$ of the total local density. This rather small value, compared to results of other $\Lambda$ CDM simulations [69], rather points towards a quiescent merger history for the galactic halo considered in our simulation. For the other velocity components and for the velocity module, we also observe strong deviations from Gaussian and Maxwellian distributions, which we parameterized by introducing generalized Gaussian and Maxwellian distributions, see Eqs. (1-2). For the velocity module, we find that $\alpha=1.5$ gives a good description of the global velocity distribution around $R_{0}=8 \mathrm{kpc}$, but a higher value $\alpha=1.95$ better accounts for the sharp drop of the high velocity tail.

The direct detection predictions are given as fits for the DAMA modulation signal and exclusion limits for null experiments. Both the elastic and the inelastic scenarios have been considered with spinindependent cross-sections. As opposed to previous works [43,60], here the regions in the parameter space are obtained directly from the velocity distributions extracted from the simulation data, without modeling. They are then compared to the predictions of various analytical models.

As the co-rotating dark disk contribution to the local density is rather small, the absolute detection rates are not significantly enhanced compared to the standard Maxwellian case. Nevertheless, the compatibility of DAMA vs. null experiments shows some improvement. For the elastic scenario, the rotating DM component causes the channeling region to enlarge outside of the XENON constraint. However, the overall goodness of fit of a global solution is still poor as most of the channeling region remains excluded. For the inelastic scenario, the situation is much brighter, as two solutions with $\delta \simeq 130 \mathrm{keV}$ are permitted. The solution with a heavy candidate $\left(M_{D M} \sim \mathrm{TeV}\right)$ becomes much more acceptable with the realistic halo provided by our simulation. Moreover, such candidate can also achieve naturally the relic abundance needed for WMAP [125], and is very little constrained by accelerator data [128]. If the DAMA modulation signal is taken seriously, all these hints concur to some interesting new physics beyond the Standard Model at the TeV scale. The solution with a mass around $M_{D M} \simeq 80 \mathrm{GeV}$ is also acceptable from both the direct detection data and the particle physics point of view. A correct relic abundance however requires some asymmetry in the dark sector [45] or some non thermal production mechanism. The accelerator constraints could be more stringent, but have to be analyzed within a particular model [129]. These questions are beyond the scope of the present paper.

\section{Acknowledgments}

We are grateful towards Jean-Charles Lambert for his help in handling GLNemo to produce the pictures of the galaxy for this paper. We would also like to thank Chiara Arina and Arman Khalatyan for helpful comments and discussions. FSL work is supported by a belgian FNRS grant and the IAP.

\section{References}

[1] Yoshiaki Sofue and Vera Rubin. Rotation Curves of Spiral Galaxies. Ann. Rev. Astron. Astrophys., 39:137-174, 2001.

[2] Albert Bosma. Dark matter in galaxies: Observational overview. Dark Matter in Galaxies, IAU symposium series, 220, 2004.

[3] F. Zwicky. Spectral displacement of extra galactic nebulae. Helv. Phys. Acta, 6:110-127, 1933.

[4] Aaron D. Lewis, David A. Buote, and John T. Stocke. Chandra Observations of Abell 2029: The Dark Matter Profile at $<0.01$ Rvir in an Unusually Relaxed Cluster. Astrophys. J., 586:135-142, 2003.

[5] Alexandre Refregier. Weak Gravitational Lensing by Large-Scale Structure. Ann. Rev. Astron. Astrophys., 41:645-668, 2003.

[6] Douglas Clowe, Anthony Gonzalez, and Maxim Markevitch. Weak lensing mass reconstruction of the interacting cluster 1e0657-558: Direct evidence for the existence of dark matter. Astrophys. J., 604:596-603, 2004.

[7] Raphael Gavazzi et al. The Sloan Lens ACS Survey. VI: Discovery and analysis of a double Einstein ring. 2008. 
[8] Shaun Cole et al. The 2dF Galaxy Redshift Survey: Power-spectrum analysis of the final dataset and cosmological implications. Mon. Not. Roy. Astron. Soc., 362:505-534, 2005.

[9] Uros Seljak et al. Cosmological parameter analysis including SDSS Ly-alpha forest and galaxy bias: Constraints on the primordial spectrum of fluctuations, neutrino mass, and dark energy. Phys. Rev., D71:103515, 2005.

[10] D. N. Spergel et al. Wilkinson microwave anisotropy probe (wmap) three year results: Implications for cosmology. 0300 .

[11] C. Amsler et al. Review of particle physics. Phys. Lett., B667:1, 2008.

[12] Jean Iliopoulos. Physics Beyond the Standard Model. 2008.

[13] Howard Baer. Physics Beyond the Standard Model. 2009.

[14] R. D. Peccei and Helen R. Quinn. Constraints Imposed by CP Conservation in the Presence of Instantons. Phys. Rev., D16:1791-1797, 1977.

[15] John R. Ellis, J. S. Hagelin, Dimitri V. Nanopoulos, Keith A. Olive, and M. Srednicki. Supersymmetric relics from the big bang. Nucl. Phys., B238:453-476, 1984.

[16] Hsin-Chia Cheng, Jonathan L. Feng, and Konstantin T. Matchev. Kaluza-Klein dark matter. Phys. Rev. Lett., 89:211301, 2002.

[17] Marco Cirelli, Nicolao Fornengo, and Alessandro Strumia. Minimal dark matter. Nucl. Phys., B753:178-194, 2006.

[18] J. Knodlseder et al. The all-sky distribution of 511-keV electron positron annihilation emission. Astron. Astrophys., 441:513-532, 2005.

[19] S. D. Hunter et al. EGRET observations of the diffuse gamma-ray emission from the galactic plane. Astrophys. J., 481:205-240, 1997.

[20] Oscar Adriani et al. An anomalous positron abundance in cosmic rays with energies 1.5.100 GeV. Nature, 458:607-609, 2009.

[21] J. Chang et al. An excess of cosmic ray electrons at energies of $300.800 \mathrm{GeV}$. Nature, 456:362-365, 2008.

[22] Celine Boehm, Dan Hooper, Joseph Silk, Michel Casse, and Jacques Paul. MeV dark matter: Has it been detected? Phys. Rev. Lett., 92:101301, 2004.

[23] W. de Boer et al. Excess of EGRET galactic gamma ray data interpreted as dark matter annihilation. 2004.

[24] J. M. Frere et al. MeV right-handed neutrinos and dark matter. Phys. Rev., D75:085017, 2007.

[25] Patrick J. Fox and Erich Poppitz. Leptophilic Dark Matter. Phys. Rev., D79:083528, 2009.

[26] Marco Cirelli, Mario Kadastik, Martti Raidal, and Alessandro Strumia. Model-independent implications of the e+, e-, anti-proton cosmic ray spectra on properties of Dark Matter. Nucl. Phys., B813:1-21, 2009.

[27] Alejandro Ibarra, David Tran, and Christoph Weniger. Decaying Dark Matter in Light of the PAMELA and Fermi LAT Data. 2009.

[28] Georg Weidenspointner et al. An asymmetric distribution of positrons in the Galactic disk revealed by $\gamma$-rays. Nature, 451:159-162, 2008.

[29] F. W. Stecker, S. D. Hunter, and D. A. Kniffen. The Likely Cause of the EGRET GeV Anomaly and its Implications. Astropart. Phys., 29:25-29, 2008.

[30] T. A. Porter and for the Fermi LAT Collaboration. Fermi LAT Measurements of the Diffuse Gamma-Ray Emission at Intermediate Galactic Latitudes. 2009.

[31] H. E. S. S. Collaboration: F. Aharonian. Probing the ATIC peak in the cosmic-ray electron spectrum with H.E.S.S. 2009.

[32] Aous A. Abdo et al. Measurement of the Cosmic Ray e+ plus e- spectrum from $20 \mathrm{GeV}$ to $1 \mathrm{TeV}$ with the Fermi Large Area Telescope. Phys. Rev. Lett., 102:181101, 2009. 
[33] Dan Hooper, Pasquale Blasi, and Pasquale Dario Serpico. Pulsars as the Sources of High Energy Cosmic Ray Positrons. JCAP, 0901:025, 2009.

[34] Dmitry Malyshev, Ilias Cholis, and Joseph Gelfand. Pulsars versus Dark Matter Interpretation of ATIC/PAMELA. 2009.

[35] Tsvi Piran, Nir J. Shaviv, and Ehud Nakar. Inhomogeneity in the Supernova Remnants as a Natural Explanation of the PAMELA/ATIC Observations. 2009.

[36] R. Bernabei et al. Search for WIMP annual modulation signature: Results from DAMA / NaI-3 and DAMA / NaI-4 and the global combined analysis. Phys. Lett., B480:23-31, 2000.

[37] Z. Ahmed et al. Search for Weakly Interacting Massive Particles with the First Five-Tower Data from the Cryogenic Dark Matter Search at the Soudan Underground Laboratory. Phys. Rev. Lett., 102:011301, 2009.

[38] J. Angle et al. First Results from the XENON10 Dark Matter Experiment at the Gran Sasso National Laboratory. Phys. Rev. Lett., 100:021303, 2008.

[39] J. Angle et al. Limits on spin-dependent WIMP-nucleon cross-sections from the XENON10 experiment. Phys. Rev. Lett., 101:091301, 2008.

[40] G. Angloher et al. Commissioning Run of the CRESST-II Dark Matter Search. 2008.

[41] R. Bernabei et al. First results from DAMA/LIBRA and the combined results with DAMA/NaI. Eur. Phys. J., C56:333-355, 2008.

[42] Frank Petriello and Kathryn M. Zurek. DAMA and WIMP dark matter. JHEP, 09:047, 2008.

[43] Malcolm Fairbairn and Thomas Schwetz. Spin-independent elastic WIMP scattering and the DAMA annual modulation signal. JCAP, 0901:037, 2009.

[44] Christopher Savage, Katherine Freese, Paolo Gondolo, and Douglas Spolyar. Compatibility of DAMA/LIBRA dark matter detection with other searches in light of new Galactic rotation velocity measurements. 2009.

[45] Chiara Arina, Fu-Sin Ling, and Michel H. G. Tytgat. The Inert Doublet Model and Inelastic Dark Matter. 2009.

[46] Ricardo A. Flores. DYNAMICAL ESTIMATES AND BOUNDS FOR THE LOCAL DENSITY OF DARK MATTER. Phys. Lett., B215:73, 1988.

[47] Y. Sofue, M. Honma, and T. Omodaka. Unified Rotation Curve of the Galaxy - Decomposition into de Vaucouleurs Bulge, Disk, Dark Halo, and the 9-kpc Rotation Dip -. 2008.

[48] Alfred Bing-Chih Chen, Phillip K. Lu, Rene A. Mendez, and William F. van Altena. Dark matter: Local volume density versus total surface density. Astron. J., 126:762, 2003.

[49] Riccardo Catena and Piero Ullio. A novel determination of the local dark matter density. 2009.

[50] J. Lavalle, Q. Yuan, D. Maurin, and X. J. Bi. Full Calculation of Clumpiness Boost factors for Antimatter Cosmic Rays in the light of LambdaCDM N-body simulation results. 2007.

[51] Mauro Sereno and Ph. Jetzer. Dark matter vs. modifications of the gravitational inverse-square law. Results from planetary motion in the solar system. Mon. Not. Roy. Astron. Soc., 371:626$632,2006$.

[52] J. M. Frere, Fu-Sin Ling, and G. Vertongen. Bound on the Dark Matter Density in the Solar System from Planetary Motions. Phys. Rev., D77:083005, 2008.

[53] Anne M. Green. The WIMP annual modulation signal and non-standard halo models. Phys. Rev., D63:043005, 2001.

[54] Anne M. Green. Effect of halo modelling on WIMP exclusion limits. Phys. Rev., D66:083003, 2002.

[55] Anne M Green. Effect of realistic astrophysical inputs on the phase and shape of the WIMP annual modulation signal. Phys. Rev., D68:023004, 2003.

[56] Christopher Savage, Katherine Freese, and Paolo Gondolo. Annual Modulation of Dark Matter in the Presence of Streams. Phys. Rev., D74:043531, 2006. 
[57] J. D. Vergados, S. H. Hansen, and O. Host. The impact of going beyond the Maxwell distribution in direct dark matter detection rates. Phys. Rev., D77:023509, 2008.

[58] J. D. Vergados. The NFW Dark Matter Density Profile Leads to Axially Symmetric Velocity Distribution-Imlications on Direct Dark Matter Searche. 2008.

[59] Marc Kamionkowski and Savvas M. Koushiappas. Galactic substructure and direct detection of dark matter. arXiv:0801.3269 [astro-ph].

[60] John March-Russell, Christopher McCabe, and Matthew McCullough. Inelastic Dark Matter, Non-Standard Halos and the DAMA/LIBRA Results. JHEP, 05:071, 2009.

[61] Simon D. M. White, Carlos S. Frenk, Marc Davis, and George Efstathiou. Clusters, filaments, and voids in a universe dominated by cold dark matter. Astrophys. J., 313:505-516, 1987.

[62] Giuseppe Tormen, Francois R. Bouchet, and S. D. M. White. The Structure and Dynamical Evolution of Dark Matter Halos. Mon. Not. Roy. Astron. Soc., 286:865-884, 1997.

[63] Romain Teyssier. Cosmological Hydrodynamics with Adaptive Mesh Refinement: a new high resolution code called RAMSES. 2001.

[64] Volker Springel. The cosmological simulation code GADGET-2. Mon. Not. Roy. Astron. Soc., 364:1105-1134, 2005.

[65] J. Diemand et al. Clumps and streams in the local dark matter distribution. 2008.

[66] Volker Springel et al. The Aquarius Project: the subhalos of galactic halos. Mon. Not. Roy. Astron. Soc., 391:1685-1711, 2008.

[67] Mark Vogelsberger et al. Phase-space structure in the local dark matter distribution and its signature in direct detection experiments. 2008.

[68] Brad K. Gibson et al. Hydrodynamical Adaptive Mesh Refinement Simulations of Disk Galaxies. 2008.

[69] J. I. Read, L. Mayer, A. M. Brooks, F. Governato, and G. Lake. A dark matter disc in three cosmological simulations of Milky Way mass galaxies. 2009.

[70] J. I. Read, G. Lake, O. Agertz, and Victor P. Debattista. Thin, thick and dark discs in LCDM. 2008.

[71] T. Bruch, J. Read, L. Baudis, and G. Lake. Detecting the Milky Way's Dark Disk. Astrophys. J., 696:920-923, 2009.

[72] Lucio Mayer, Fabio Governato, and Tobias Kaufmann. The formation of disk galaxies in computer simulations. 2008.

[73] Fabio Governato et al. Forming Disk Galaxies in Lambda CDM Simulations. Mon. Not. Roy. Astron. Soc., 374:1479-1494, 2007.

[74] Fabio Governato et al. Forming a Large Disk Galaxy from a $\mathrm{z}<1$ Major Merger. 2008.

[75] R. Teyssier. Cosmological hydrodynamics with adaptive mesh refinement. a new high resolution code called ramses. A\&SA, 385:337-364, 2002.

[76] Neal Katz, Lars Hernquist, and David H. Weinberg. Galaxies and gas in a cold dark matter universe. Astrophys. J., 399:L109, 1992.

[77] Ralph S. Sutherland and Michael A. Dopita. Cooling functions for low - density astrophysical plasmas. Astrophys. J. Suppl., 88:253, 1993.

[78] Yann Rasera and Romain Teyssier. The History of the Baryon Budget: Cosmic Logistics in a Hierarchical Universe. 2005.

[79] Y. Dubois and R. Teyssier. Cosmological MHD simulation of a cooling flow cluster. 2008.

[80] Wayne Hu and Daniel J. Eisenstein. The Structure of structure formation theories. Phys. Rev., D59:083509, 1999.

[81] E. Athanassoula. On the nature of bulges in general and of box/peanut bulges in particular. Input from N-body simulations. Mon. Not. Roy. Astron. Soc., 358:1477-1488, 2005. 
[82] Ian R. Walker, J. Christopher Mihos, and Lars Hernquist. Quantifying the Fragility of Galactic Disks in Minor Mergers. Astrophys. J., 460:121, 1996.

[83] Jorge Penarrubia, Alan McConnachie, and Arif Babul. On the formation of extended galactic disks by tidally disrupted dwarf galaxies. Astrophys. J., 650:L33-L36, 2006.

[84] Stelios Kazantzidis, James S. Bullock, Andrew R. Zentner, Andrey V. Kravtsov, and Leonidas A. Moustakas. Cold Dark Matter Substructure and Galactic Disks I: Morphological Signatures of Hierarchical Satellite Accretion. 2007.

[85] G. Gilmore and N. Reid. New light on faint stars. III - Galactic structure towards the South Pole and the Galactic thick disc. Mon. Not. Roy. Astron. Soc., 202:1025-1047, 1983.

[86] Chris W. Purcell, James S. Bullock, and Manoj Kaplinghat. The Dark Disk of the Milky Way. 2009.

[87] Mario Juric et al. The Milky Way Tomography with SDSS. 1. Stellar Number Density Distribution. Astrophys. J., 673:864-914, 2008.

[88] C. Soubiran, O. Bienayme, and A. Siebert. Vertical distribution of Galactic disk stars I - Kinematics and metallicity. Astron. Astrophys., 398:141-152, 2003.

[89] B. Nordstrom et al. The Geneva-Copenhagen survey of the Solar neighbourhood: Ages, metallicities, and kinematic properties of 14,000 F and G dwarfs. Astron. Astrophys., 418:989, 2004.

[90] Graciela B. Gelmini. Search for Dark Matter. Int. J. Mod. Phys., A23:4273-4288, 2008.

[91] Dan Hooper. TASI 2008 Lectures on Dark Matter. 2009.

[92] R. Bernabei et al. Possible implications of the channeling effect in NaI(Tl) crystals. Eur. Phys. J., C53:205-213, 2008.

[93] A. Bottino, F. Donato, N. Fornengo, and S. Scopel. Zooming in on light relic neutralinos by direct detection and measurements of galactic antimatter. Phys. Rev., D77:015002, 2008.

[94] J. D. Lewin and P. F. Smith. Review of mathematics, numerical factors, and corrections for dark matter experiments based on elastic nuclear recoil. Astropart. Phys., 6:87-112, 1996.

[95] Sarah Andreas, Thomas Hambye, and Michel H. G. Tytgat. WIMP dark matter, Higgs exchange and DAMA. JCAP, 0810:034, 2008.

[96] Graciela Gelmini and Paolo Gondolo. DAMA dark matter detection compatible with other searches. 2004.

[97] Paolo Gondolo and Graciela Gelmini. Compatibility of DAMA dark matter detection with other searches. Phys. Rev., D71:123520, 2005.

[98] A. Bottino, N. Fornengo, and S. Scopel. Light relic neutralinos. Phys. Rev., D67:063519, 2003.

[99] A. Bottino, F. Donato, N. Fornengo, and S. Scopel. Lower bound on the neutralino mass from new data on CMB and implications for relic neutralinos. Phys. Rev., D68:043506, 2003.

[100] A. Bottino, F. Donato, N. Fornengo, and S. Scopel. Light neutralinos and WIMP direct searches. Phys. Rev., D69:037302, 2004.

[101] David Tucker-Smith and Neal Weiner. Inelastic dark matter. Phys. Rev., D64:043502, 2001.

[102] David Tucker-Smith and Neal Weiner. The status of inelastic dark matter. Phys. Rev., D72:063509, 2005.

[103] Spencer Chang, Graham D. Kribs, David Tucker-Smith, and Neal Weiner. Inelastic Dark Matter in Light of DAMA/LIBRA. Phys. Rev., D79:043513, 2009.

[104] Yanou Cui, David E. Morrissey, David Poland, and Lisa Randall. Candidates for Inelastic Dark Matter. JHEP, 05:076, 2009.

[105] Douglas P. Finkbeiner, Tongyan Lin, and Neal Weiner. Inelastic Dark Matter and DAMA/LIBRA: An Experimentum Crucis. 2009.

[106] Graciela Gelmini and Paolo Gondolo. WIMP annual modulation with opposite phase in late-infall halo models. Phys. Rev., D64:023504, 2001. 
[107] Fu-Sin Ling, Pierre Sikivie, and Stuart Wick. Diurnal and Annual Modulation of Cold Dark Matter Signals. Phys. Rev., D70:123503, 2004.

[108] Martin C. Smith et al. The RAVE Survey: Constraining the Local Galactic Escape Speed. Mon. Not. Roy. Astron. Soc., 379:755-772, 2007.

[109] A. K. Drukier, K. Freese, and D. N. Spergel. Detecting Cold Dark Matter Candidates. Phys. Rev., D33:3495-3508, 1986.

[110] Katherine Freese, Joshua A. Frieman, and Andrew Gould. Signal Modulation in Cold Dark Matter Detection. Phys. Rev., D37:3388, 1988.

[111] Richard H. Helm. Inelastic and Elastic Scattering of 187-Mev Electrons from Selected Even-Even Nuclei. Phys. Rev., 104:1466-1475, 1956.

[112] Gintaras Duda, Ann Kemper, and Paolo Gondolo. Model independent form factors for spin independent neutralino nucleon scattering from elastic electron scattering data. JCAP, 0704:012, 2007.

[113] Christopher Savage, Graciela Gelmini, Paolo Gondolo, and Katherine Freese. Compatibility of DAMA/LIBRA dark matter detection with other searches. JCAP, 0904:010, 2009.

[114] Walter Dehnen and James Binney. Local stellar kinematics from Hipparcos data. Mon. Not. Roy. Astron. Soc., 298:387-394, 1998.

[115] M. J. Reid et al. Trigonometric Parallaxes of Massive Star Forming Regions: VI. Galactic Structure, Fundamental Parameters and Non- Circular Motions. Astrophys. J., 700:137-148, 2009.

[116] A. Bottino, F. Donato, N. Fornengo, and S. Scopel. Interpreting the recent results on direct search for dark matter particles in terms of relic neutralino. Phys. Rev., D78:083520, 2008.

[117] Spencer Chang, Aaron Pierce, and Neal Weiner. Using the Energy Spectrum at DAMA/LIBRA to Probe Light Dark Matter. 2008.

[118] D. S. Akerib et al. First results from the cryogenic dark matter search in the Soudan Underground Lab. Phys. Rev. Lett., 93:211301, 2004.

[119] D. S. Akerib et al. Limits on spin-independent WIMP nucleon interactions from the two-tower run of the Cryogenic Dark Matter Search. Phys. Rev. Lett., 96:011302, 2006.

[120] D. S. Akerib et al. New results from the Cryogenic Dark Matter Search experiment. Phys. Rev., D68:082002, 2003.

[121] G. Angloher et al. Limits on WIMP dark matter using sapphire cryogenic detectors. Astropart. Phys., 18:43-55, 2002.

[122] G. Angloher et al. Limits on WIMP dark matter using scintillating CaWO-4 cryogenic detectors with active background suppression. Astropart. Phys., 23:325-339, 2005.

[123] Fu-Sin Ling. in preparation.

[124] G. Hinshaw et al. Five-Year Wilkinson Microwave Anisotropy Probe (WMAP) Observations:Data Processing, Sky Maps, \& Basic Results. 2008.

[125] T. Hambye, F. S. Ling, L. Lopez Honorez, and J. Rocher. Scalar Multiplet Dark Matter. 2009.

[126] E. Athanassoula, F. S. Ling, E. Nezri, and R. Teyssier. Gamma ray fluxes from a cosmological dark matter simulation. Astropart. Phys., 31:37-45, 2009.

[127] Jurg Diemand and Ben Moore. The structure and evolution of cold dark matter halos. 2009.

[128] Riccardo Barbieri, Lawrence J. Hall, and Vyacheslav S. Rychkov. Improved naturalness with a heavy Higgs: An alternative road to LHC physics. Phys. Rev., D74:015007, 2006.

[129] Erik Lundstrom, Michael Gustafsson, and Joakim Edsjo. The Inert Doublet Model and LEP II Limits. 2008. 

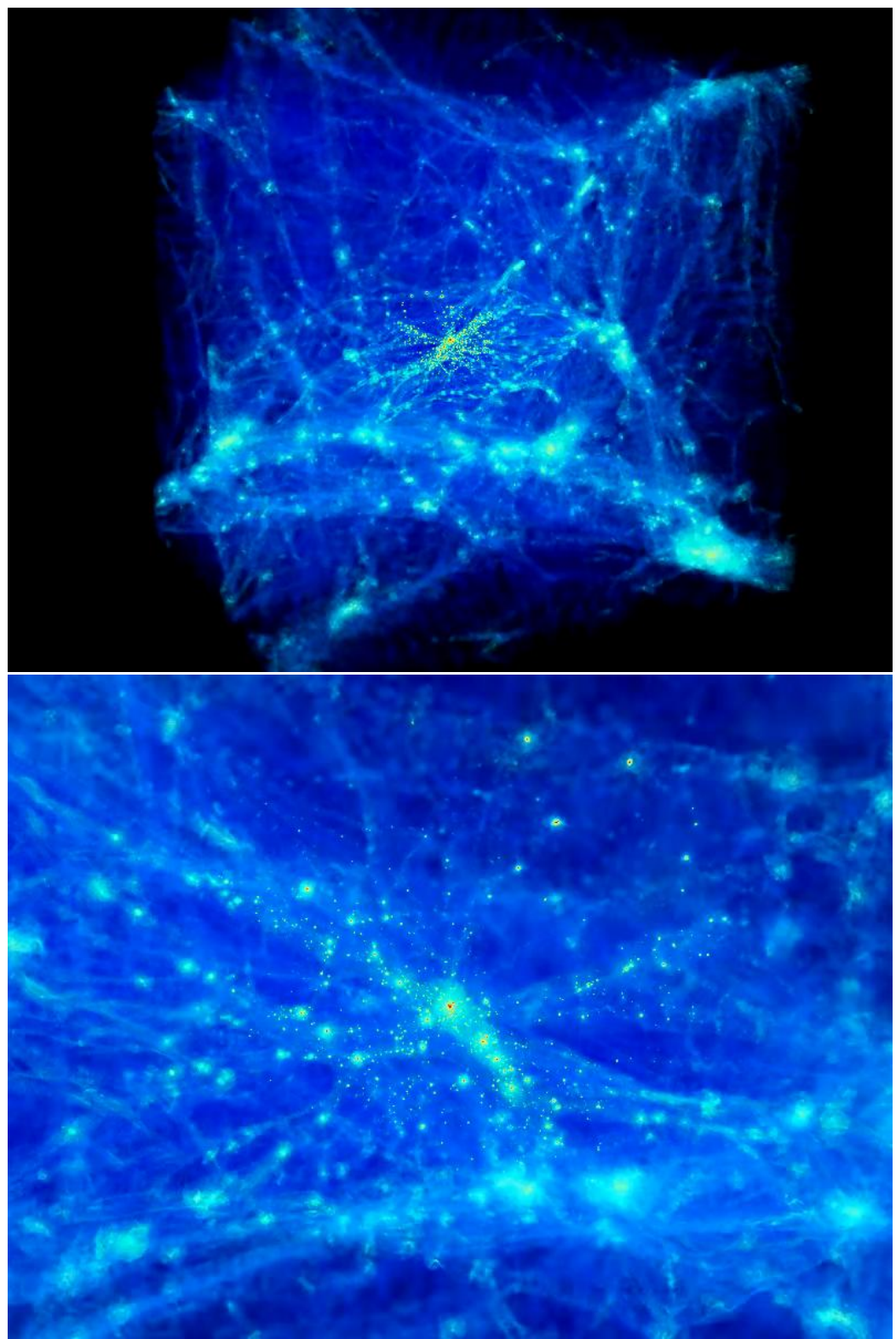

Figure 1: View of the simulation box (top), with a zoom on the central and better resolved region (bottom). 


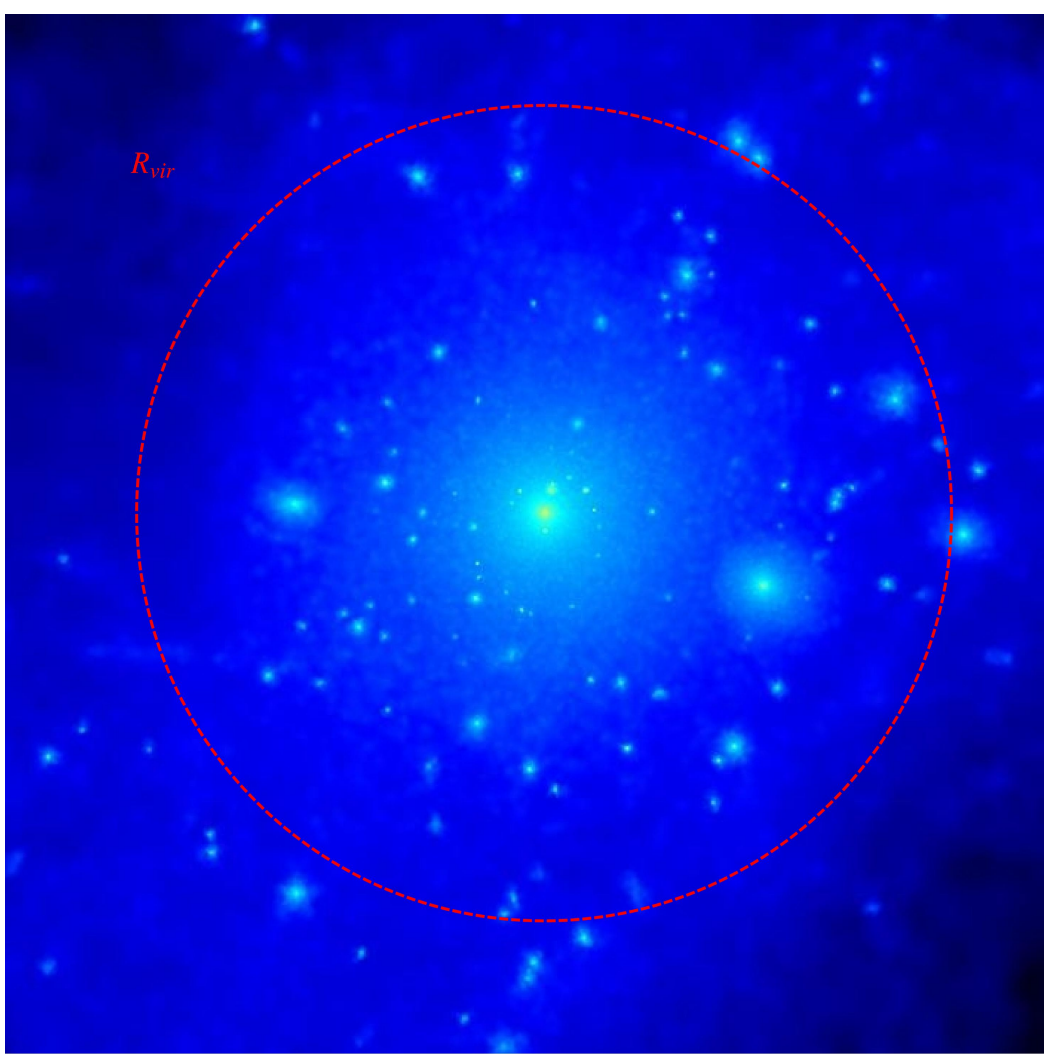

Figure 2: View of the dark matter halo of the central galactic object of Fig. 1, with its virial radius. 


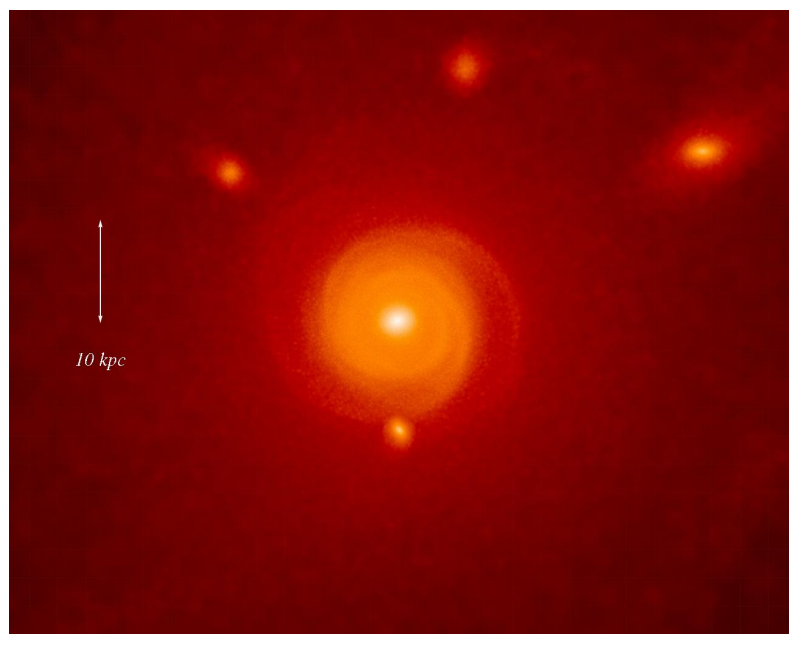

a) Stars face on

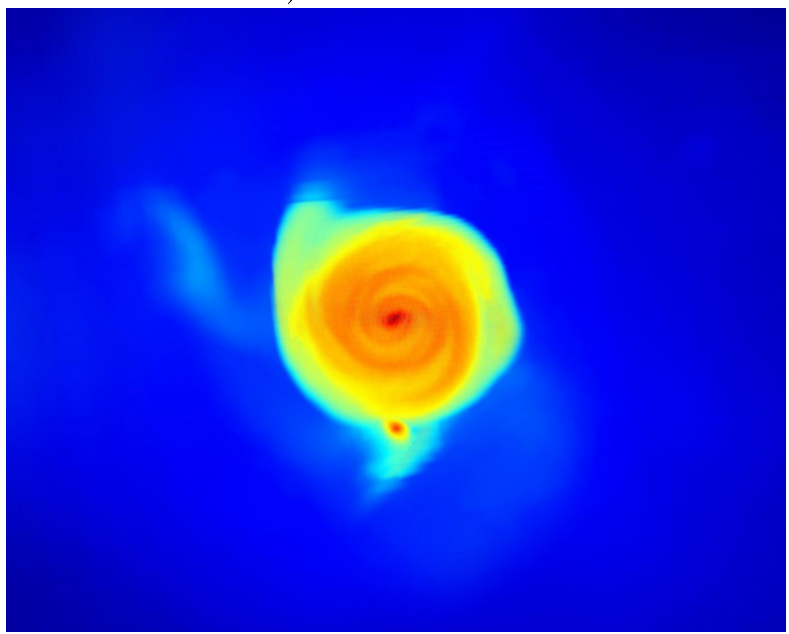

c) Gas face on

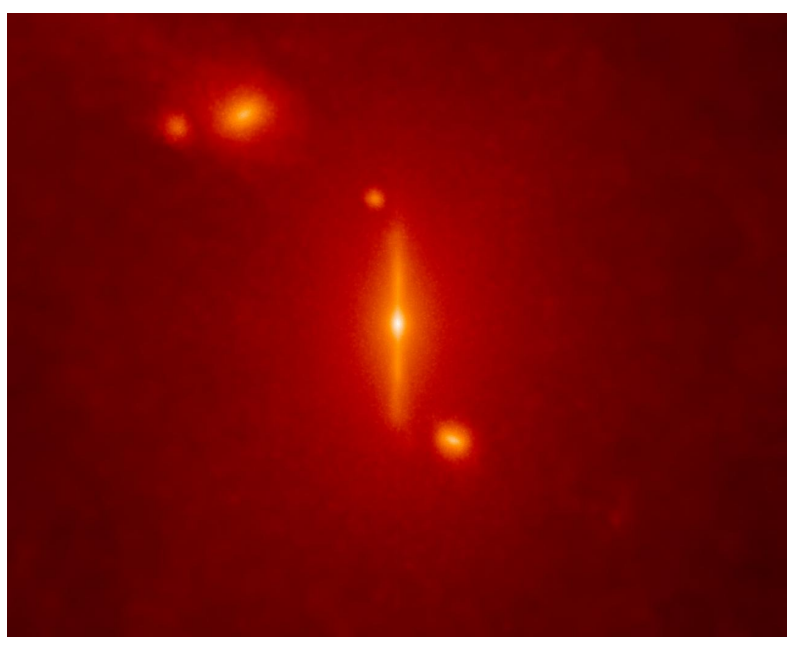

b) Stars edge on

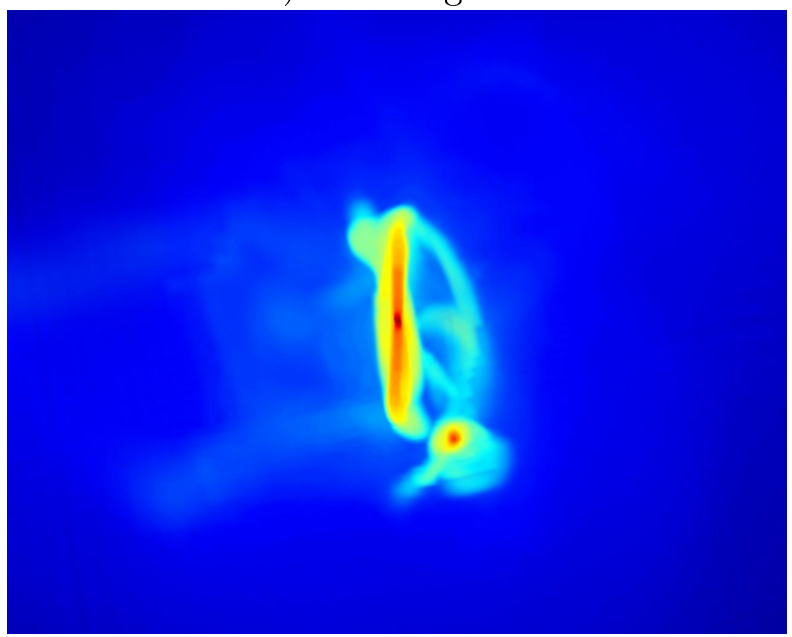

d) Gas edge on

Figure 3: Views of the stars and gas distribution of the galactic structure considered in this paper. 

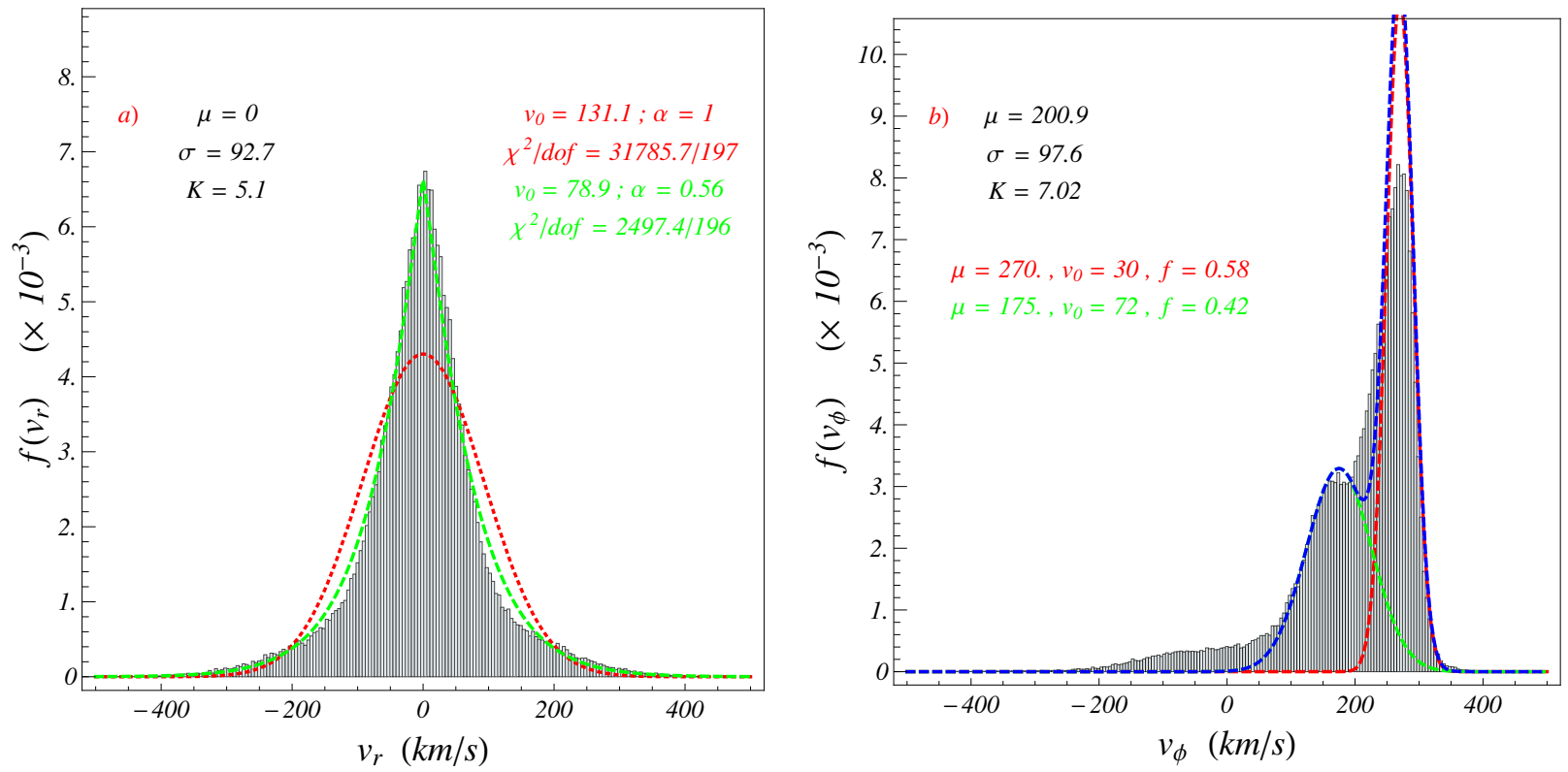

Figure 4: Velocity distributions of star particles $\left(N_{\text {star }}=143,320\right)$ in a ring $7<R<9 k p c,|z|<3$ kpc around the galactic plane.

a) Radial velocity $v_{r}$ with Gaussian (red) and generalized Gaussian (green) fits (cfr. Eq. (1)).

b) Tangential velocity $v_{\phi}$ with a double Gaussian fit, with a thin (red) and a thick (green) disk components. $f$ indicates the fraction for each component.

$\mu, \sigma$ (both in $\mathrm{km} / \mathrm{s}$ ) and $K$ stand for the mean, the standard deviation and the Kurtosis parameter of the distribution. The goodness of fit is indicated by the value of the $\chi^{2}$ vs. the number of degrees of freedom (dof). 

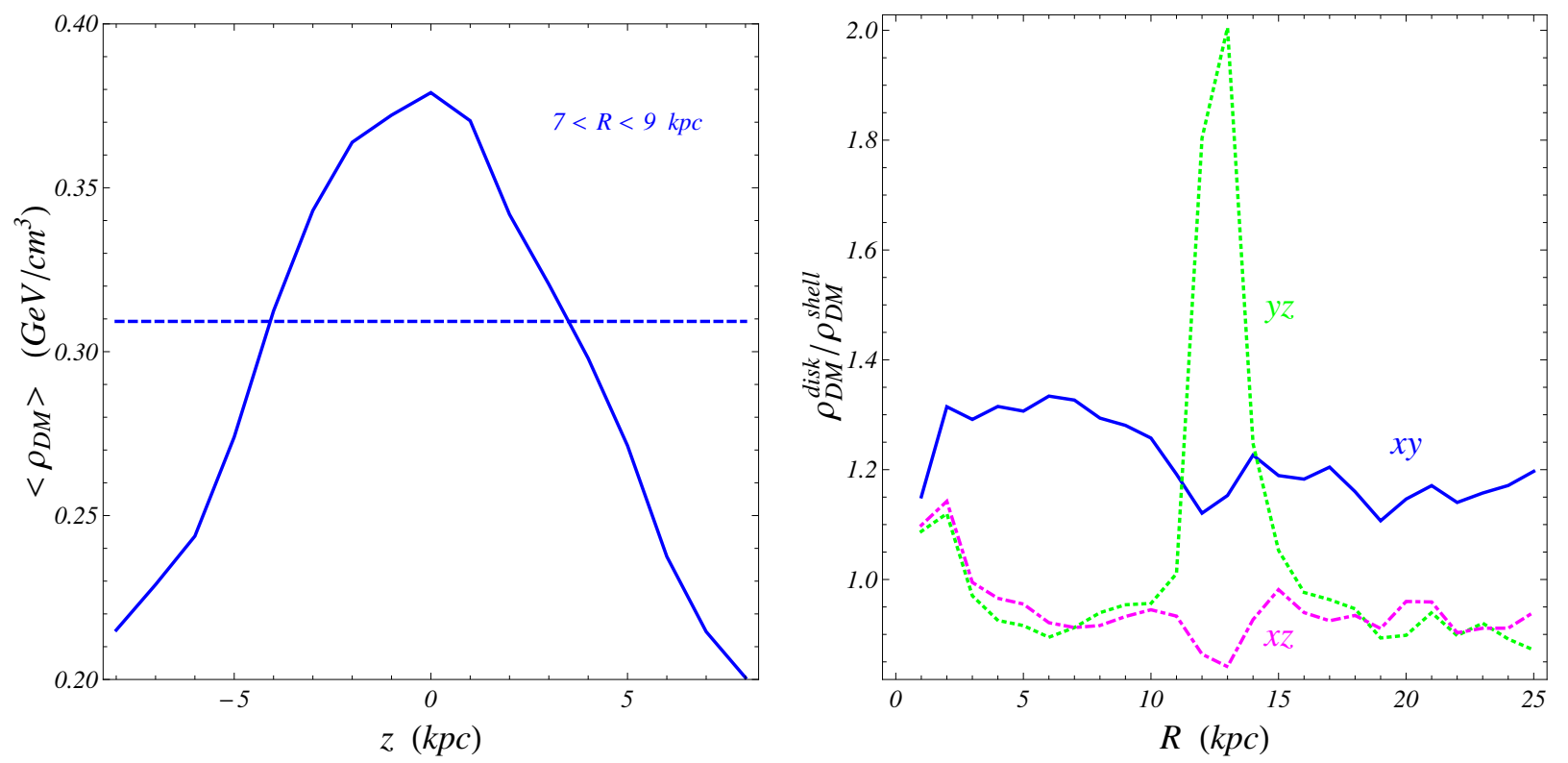

Figure 5: (Left) Average density of DM particles with $7<R<9 k p c$ as a function of the height from the galactic disk $z$ ( $R$ is the spherical radius to the galactic center). The dashed line gives the average value for the entire spherical shell. To select particles in $z$ slices, we used a thickness $\delta z=2 \mathrm{kpc}$.

(Right) Ratio of ring to shell densities as a function of distance from the galactic center for different planes. The ratio fluctuates around 1.2 for the galactic plane (blue), while it drops to a value $\sim 0.9$ for other planes (green, magenta). For the plane $y z$, the sudden peak at $R \simeq 13 \mathrm{kpc}$ is due to the presence of a satellite halo, visible on Fig. 6.b.
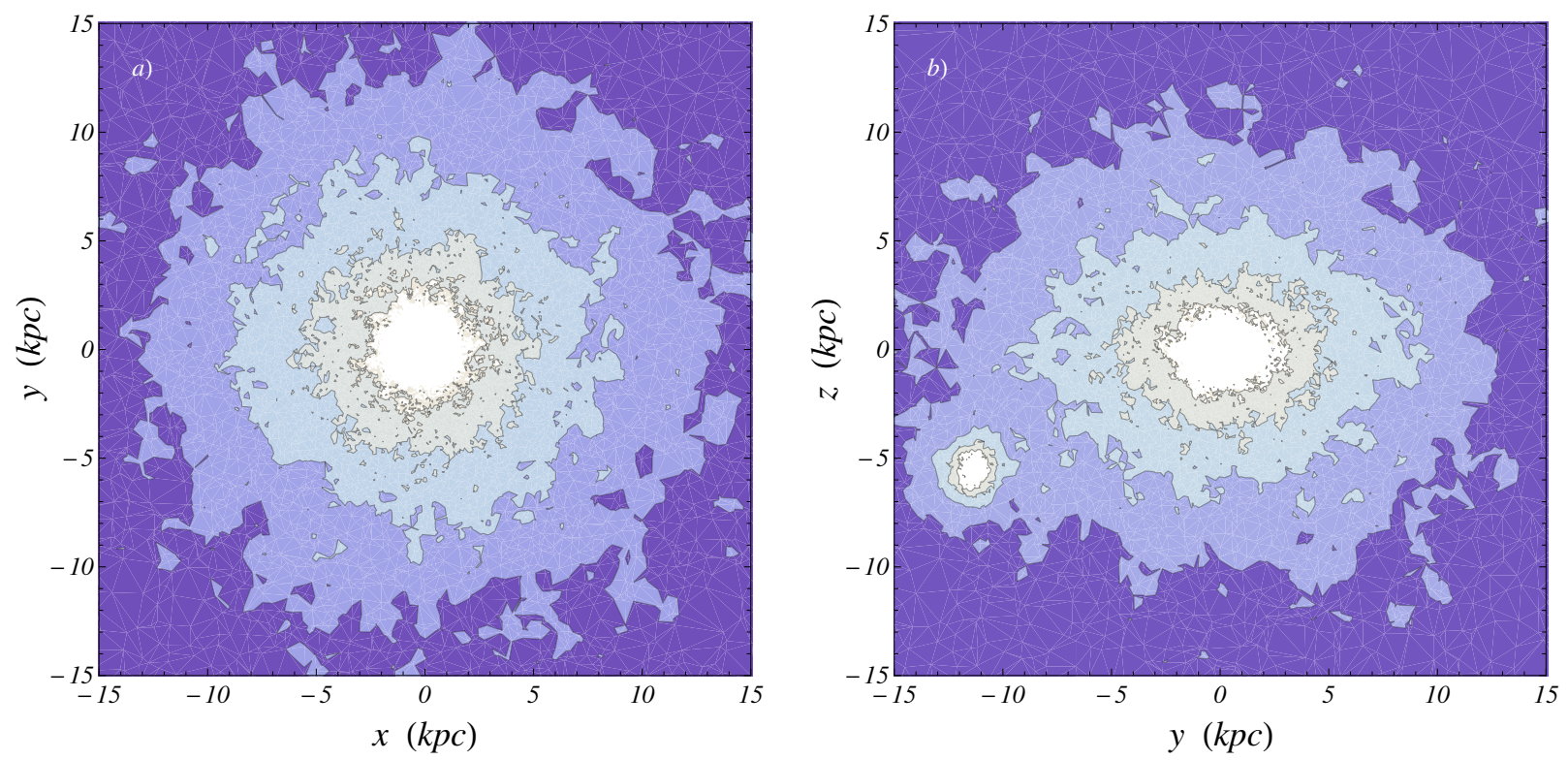

Figure 6: Density maps of the dark matter halo in the planes a) xy (galactic plane), b) yz. Contours correspond to $\rho_{D M}=\{0.1,0.3,1.0,3.0\} \mathrm{GeV} / \mathrm{cm}^{3}$. 

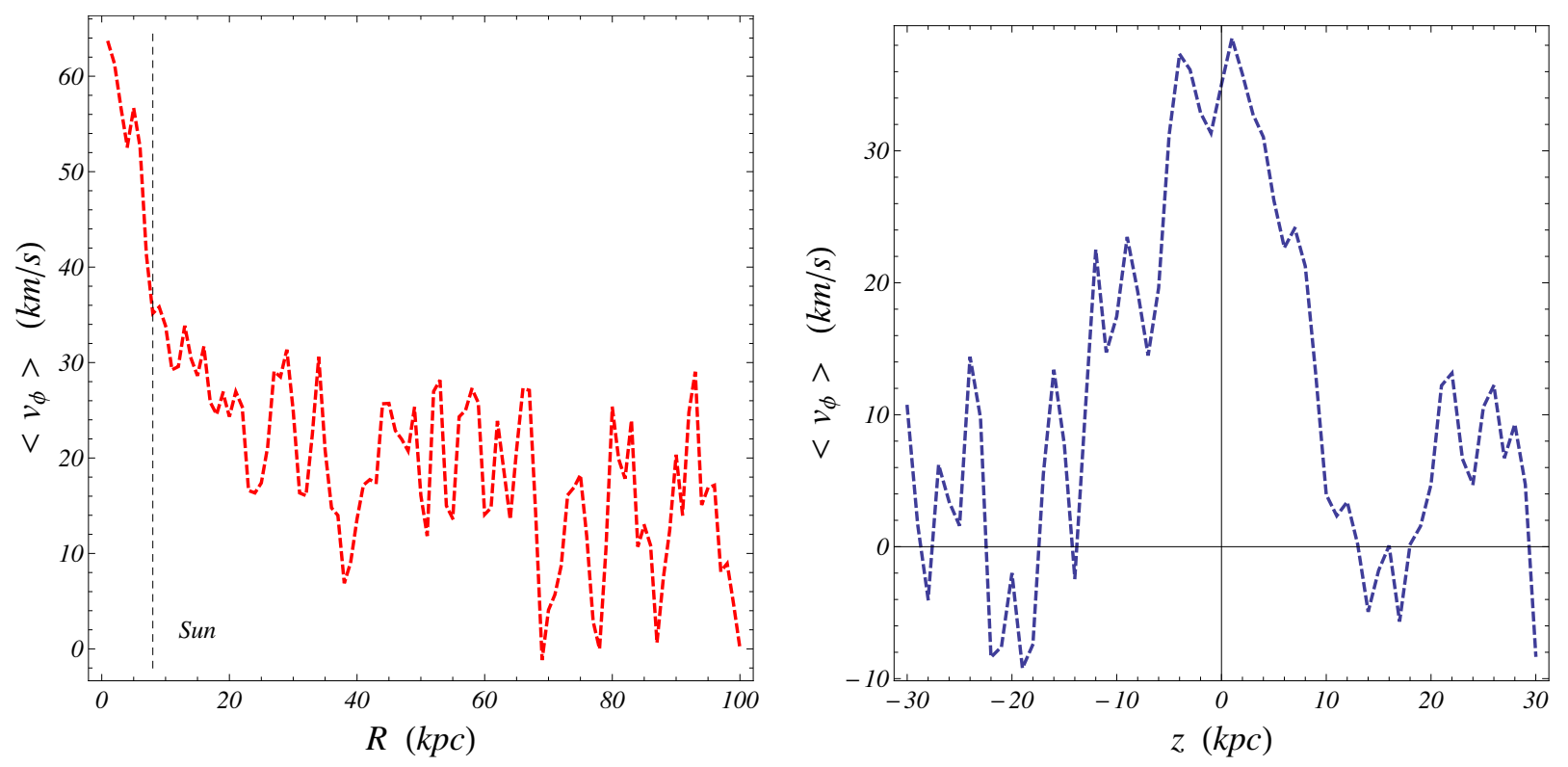

Figure 7: Mean tangential velocity $\left\langle v_{\phi}\right\rangle$ as a function of $R$ for $z=0$ (left panel), and as a function of $z$ for $r=8 \mathrm{kpc}$ (right), where $r=\sqrt{x^{2}+y^{2}}$ is the polar radius.

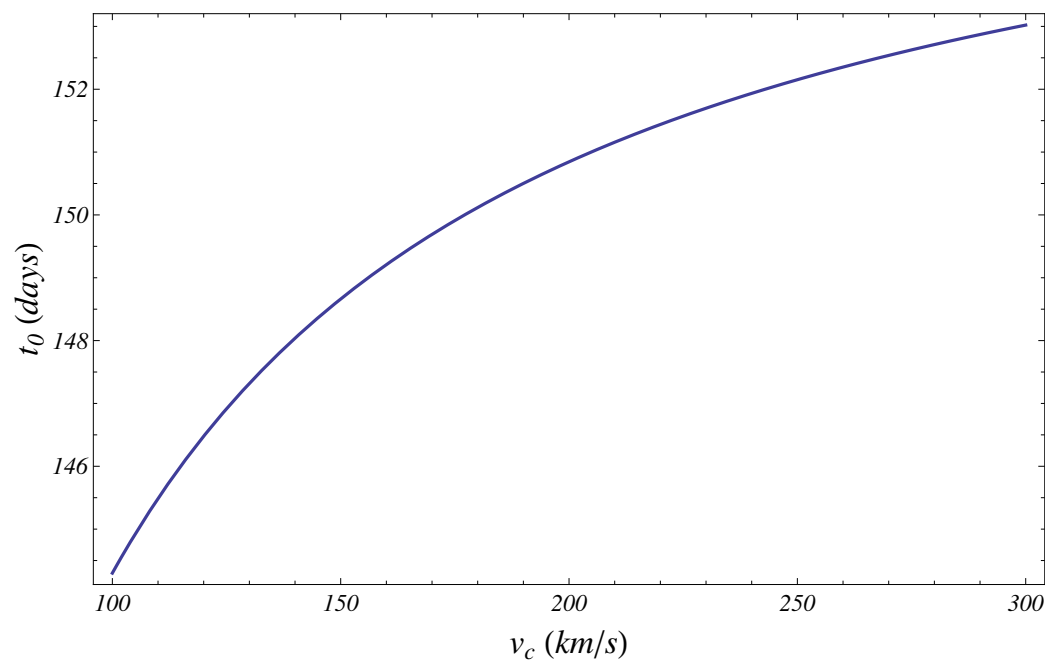

Figure 8: Variation of the phase $t_{0}$ corresponding to a maximal Earth velocity and event rate as a function of the galactic circular velocity $v_{c}$. 


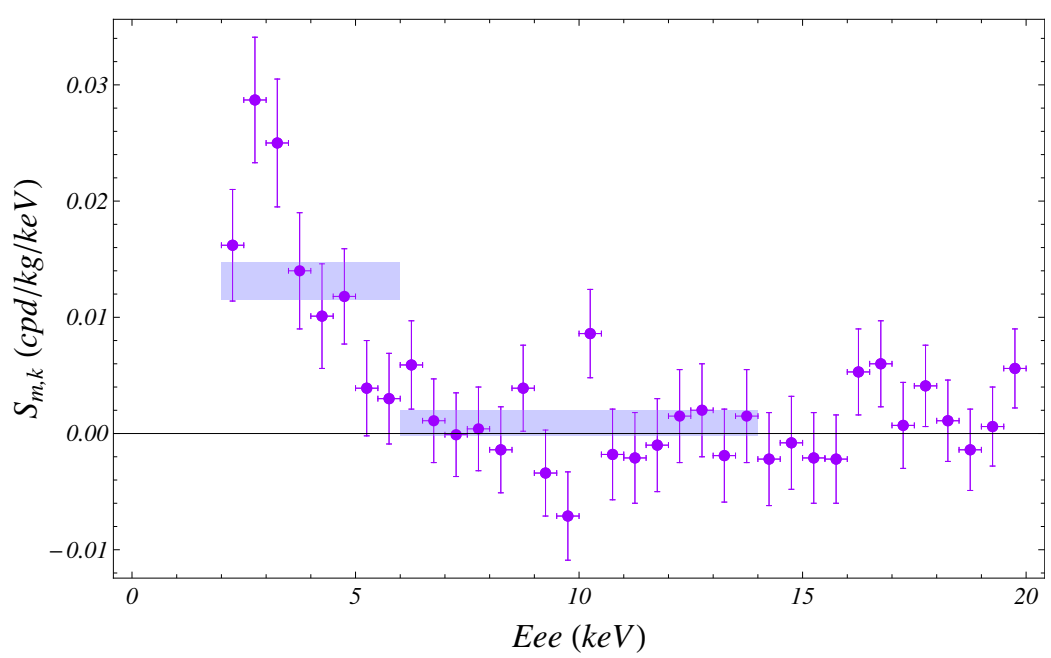

Figure 9: DAMA modulation amplitude data (in counts per day per $\mathrm{kg}$ per $\mathrm{keV}$ ) as a function of the recoil energy, expressed in electron-equivalent $\mathrm{keV}$ units. The $36 \mathrm{bins}$, from 2 to $20 \mathrm{keV}$, show a modulation signal in the low energy part, from 2 to $6 \mathrm{keV}$. The upper part is compatible with zero modulation, as shown in the two bins version of the data (from 2 to $6 \mathrm{keV}$ and from 6 to $14 \mathrm{keV}$ ).
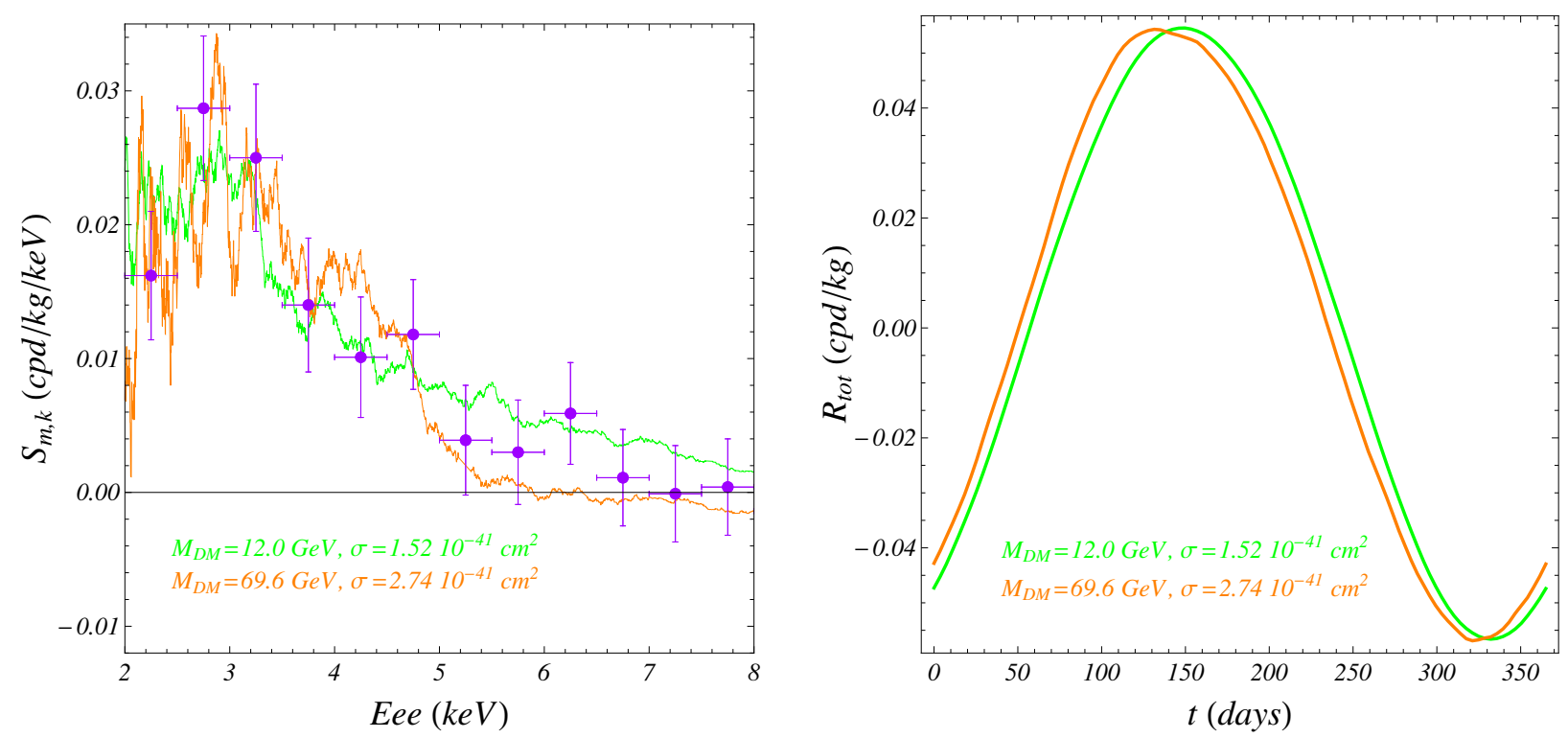

Figure 10: Elastic Scenario Modulation amplitude as a function of recoil energy (left) or time (right) for the best-fit points of Fig. 14. In the case of the time variation, events with a recoil energy $2 \leq E_{R} \leq 6 \mathrm{keV}$ (in electron-equivalent units) were considered. 

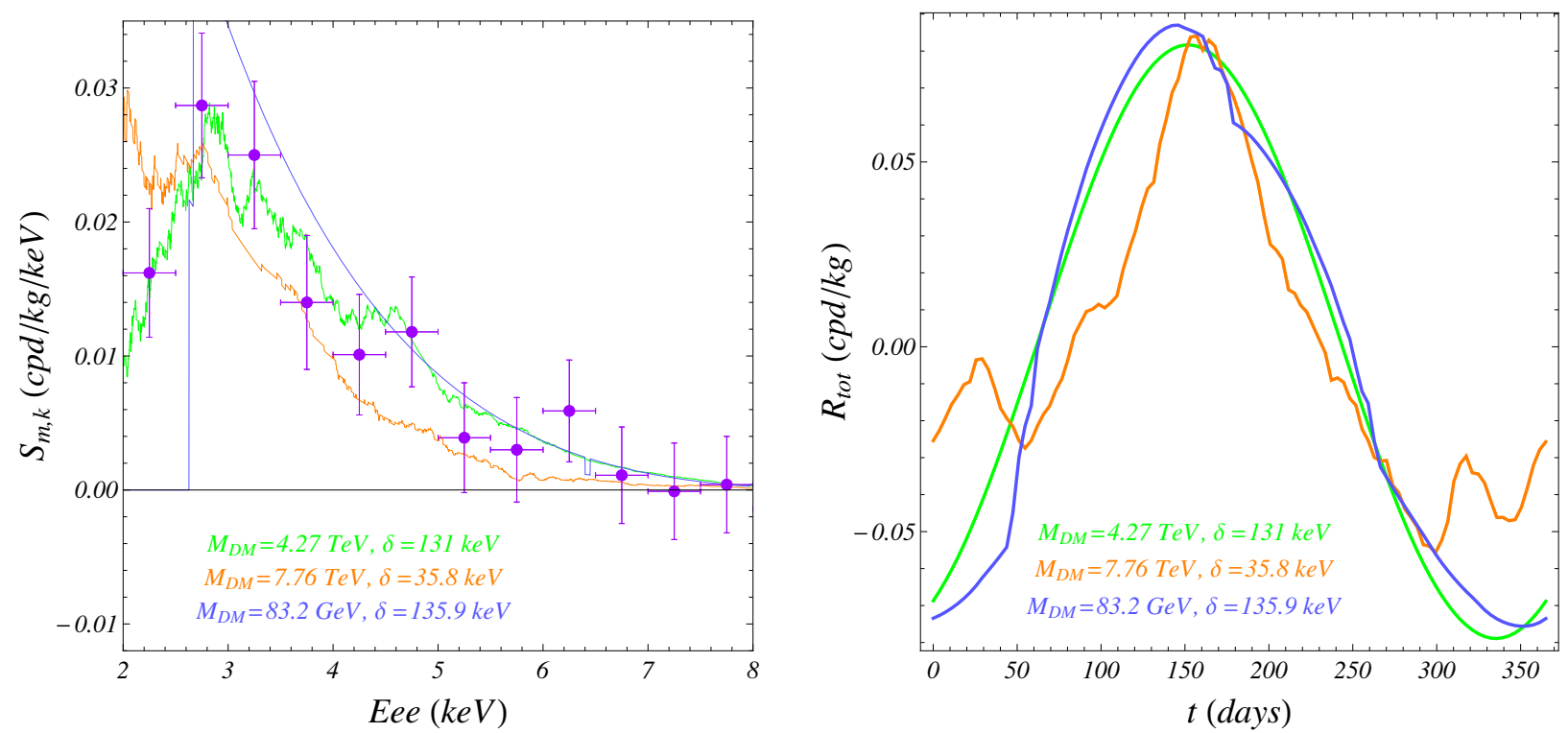

Figure 11: Inelastic Scenario Modulation amplitude as a function of recoil energy (left) or time (right) for the best-fit points of Fig. 15. In the case of the time variation, events with a recoil energy $2 \leq E_{R} \leq 6 \mathrm{keV}$ (in electron-equivalent units) were considered. 

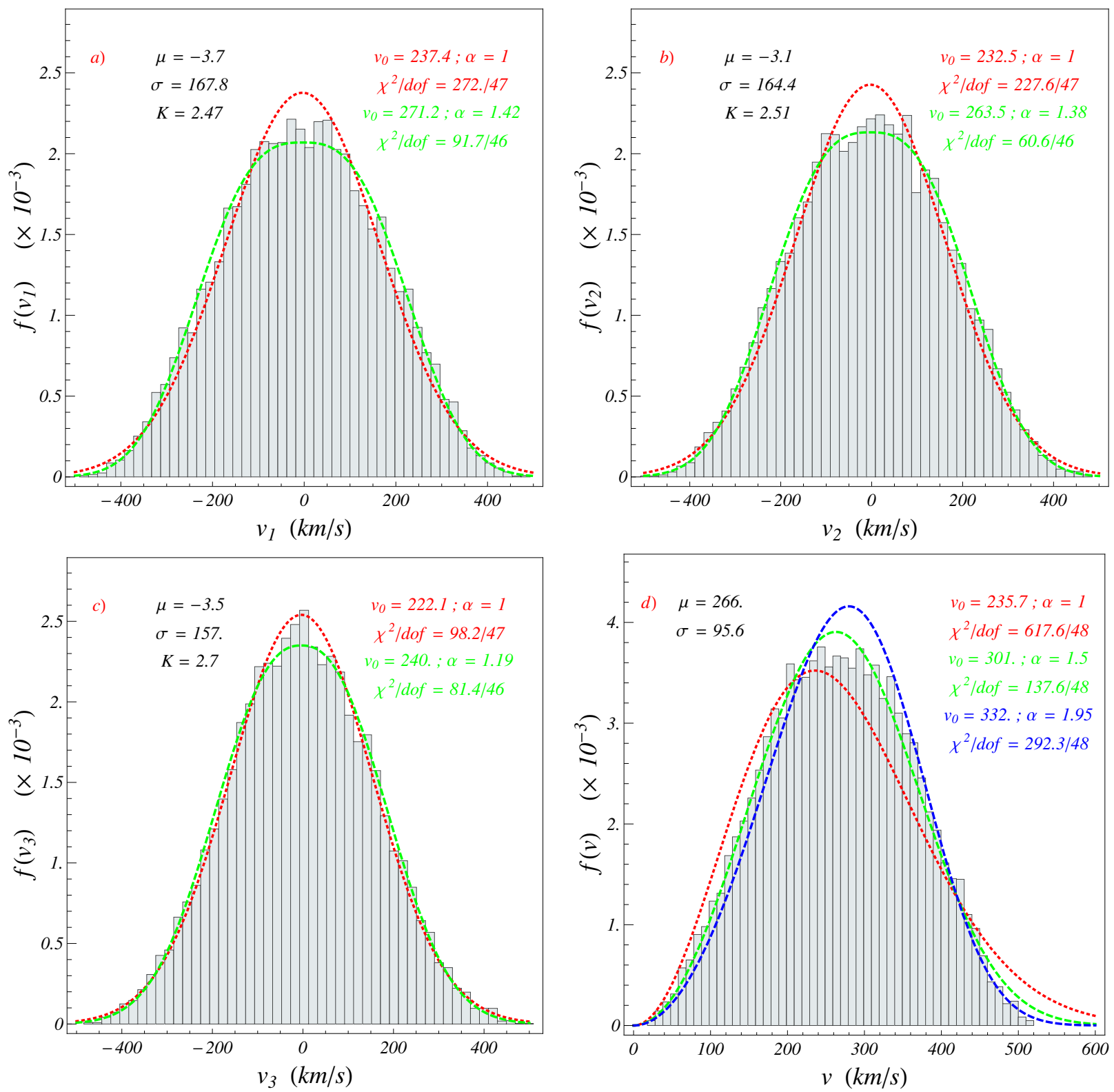

Figure 12: Velocity distributions of dark matter particles $\left(N_{\text {shell }}=16,545\right)$ in a spherical shell $7<R<9 k p c$ around the galactic center.

a), b) and c) Velocity components along the principal axes of the velocity dispersion tensor, together with the Gaussian (red) and a generalized Gaussian (green) distribution fits (cfr. Eq. (1)).

d) Velocity module, with a Maxwellian (red) and two generalized Maxwellian (green and blue) fits (cfr. Eq. (2)). $\mu, \sigma$ (both in $\mathrm{km} / \mathrm{s}$ ) and $K$ stand for the mean, the standard deviation and the Kurtosis parameter of the distribution. The goodness of fit is indicated by the value of the $\chi^{2}$ vs. the number of degrees of freedom (dof). 

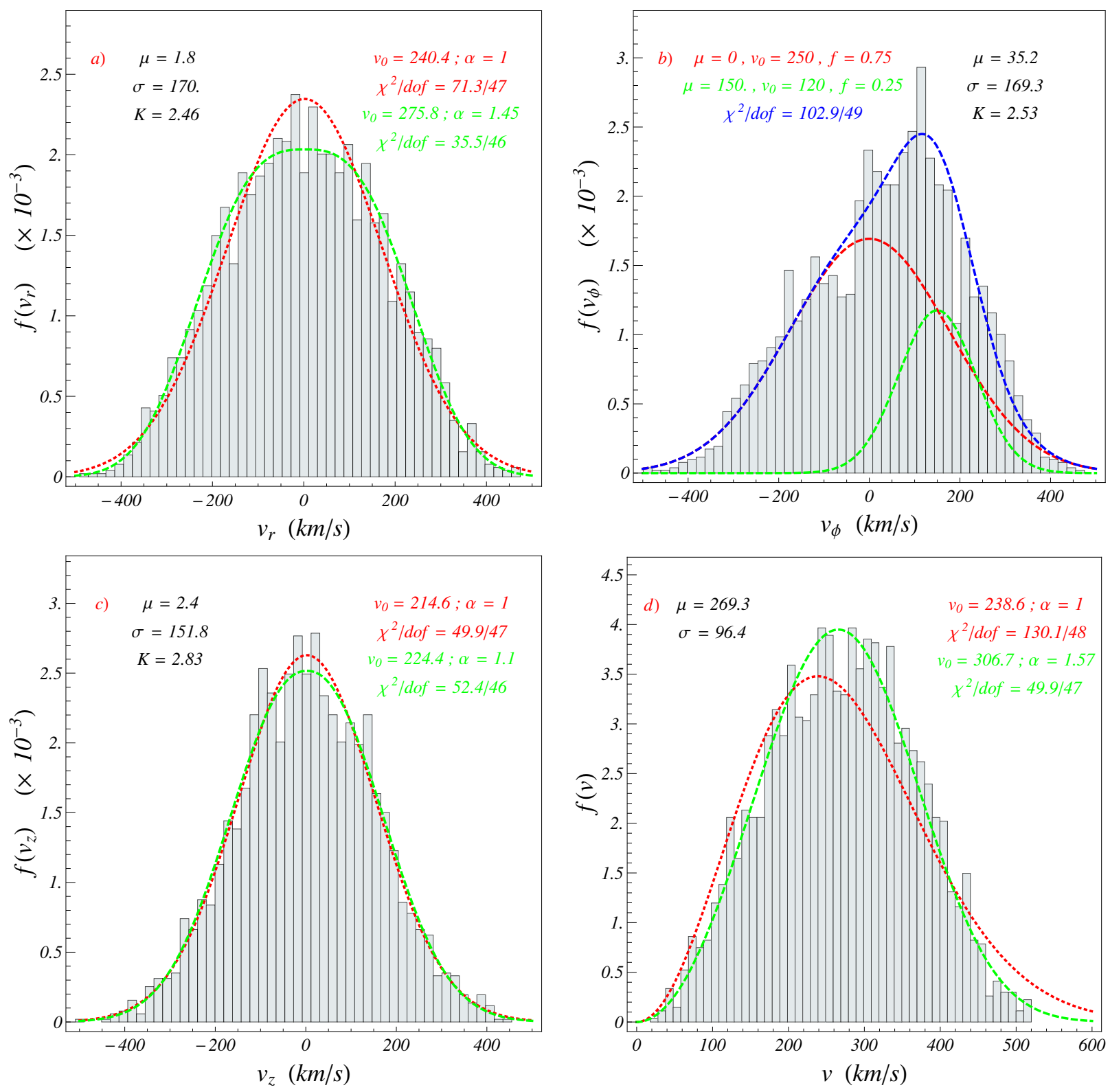

Figure 13: Velocity distributions of dark matter particles $\left(N_{\text {ring }}=2,662\right)$ in a ring $7<R<9 k p c,|z|<1 k p c$ around the galactic plane.

a) Radial velocity v $v_{r}$, with Gaussian (red) and generalized Gaussian (green) fits (cfr. Eq. (1)).

b) Tangential velocity $v_{\phi}$, with a double Gaussian fit. $f$ indicates the fraction of each component.

c) Velocity across the galactic plane $v_{z}$, with Gaussian (red) and generalized Gaussian (green) fits (cfr. Eq. (1)).

d) Velocity module, with a Maxwellian (red) and generalized Maxwellian (green) fits (cfr. Eq. (2)).

$\mu, \sigma$ (both in $\mathrm{km} / \mathrm{s}$ ) and $K$ stand for the mean, the standard deviation and the Kurtosis parameter of the distribution. The goodness of fit is indicated by the value of the $\chi^{2}$ vs. the number of degrees of freedom (dof). 

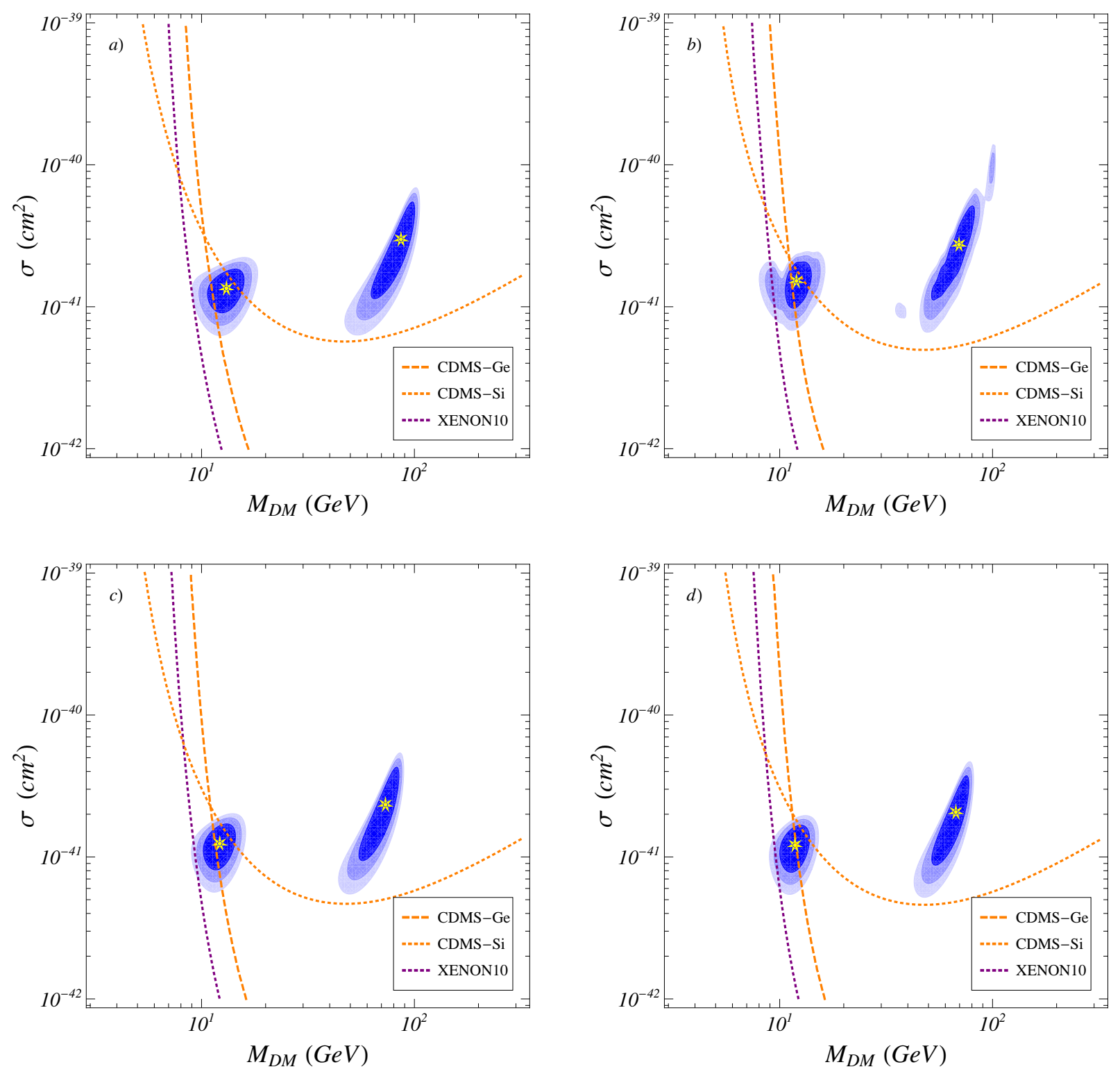

Figure 14: Elastic Scenario, allowed regions in the plane $M_{D M}-\sigma$

a) Standard Maxwellian halo $\left(\rho_{D M}=0.3 \mathrm{GeV} / \mathrm{cm}^{3}, v_{c}=220 \mathrm{~km} / \mathrm{s}, v_{0}=220 \mathrm{~km} / \mathrm{s}, v_{\text {esc }}=600 \mathrm{~km} / \mathrm{s}\right.$ )

b) $N$-body simulation with baryons $\left(\rho_{D M}=0.37 \mathrm{GeV} / \mathrm{cm}^{3}, v_{c}=220 \mathrm{~km} / \mathrm{s}\right)$

c) Generalized Maxwellian halo $\left(\rho_{D M}=0.37 \mathrm{GeV} / \mathrm{cm}^{3}, v_{c}=190 \mathrm{~km} / \mathrm{s}, v_{0}=301 \mathrm{~km} / \mathrm{s}, \alpha=1.5\right.$ )

d) Generalized Maxwellian halo ( $\rho_{D M}=0.37 \mathrm{GeV} / \mathrm{cm}^{3}, v_{c}=190 \mathrm{~km} / \mathrm{s}, v_{0}=332 \mathrm{~km} / \mathrm{s}, \alpha=1.95$ )

For c) and d), the circular velocity $v_{c}$ has been reduced compared to the standard value in order to mimic a halo rotation. DAMA contours correspond to 90, 99 and $99.9 \%$ CL. Stars indicate local best-fit points. All other exclusion curves are at the $99.9 \%$ CL. 

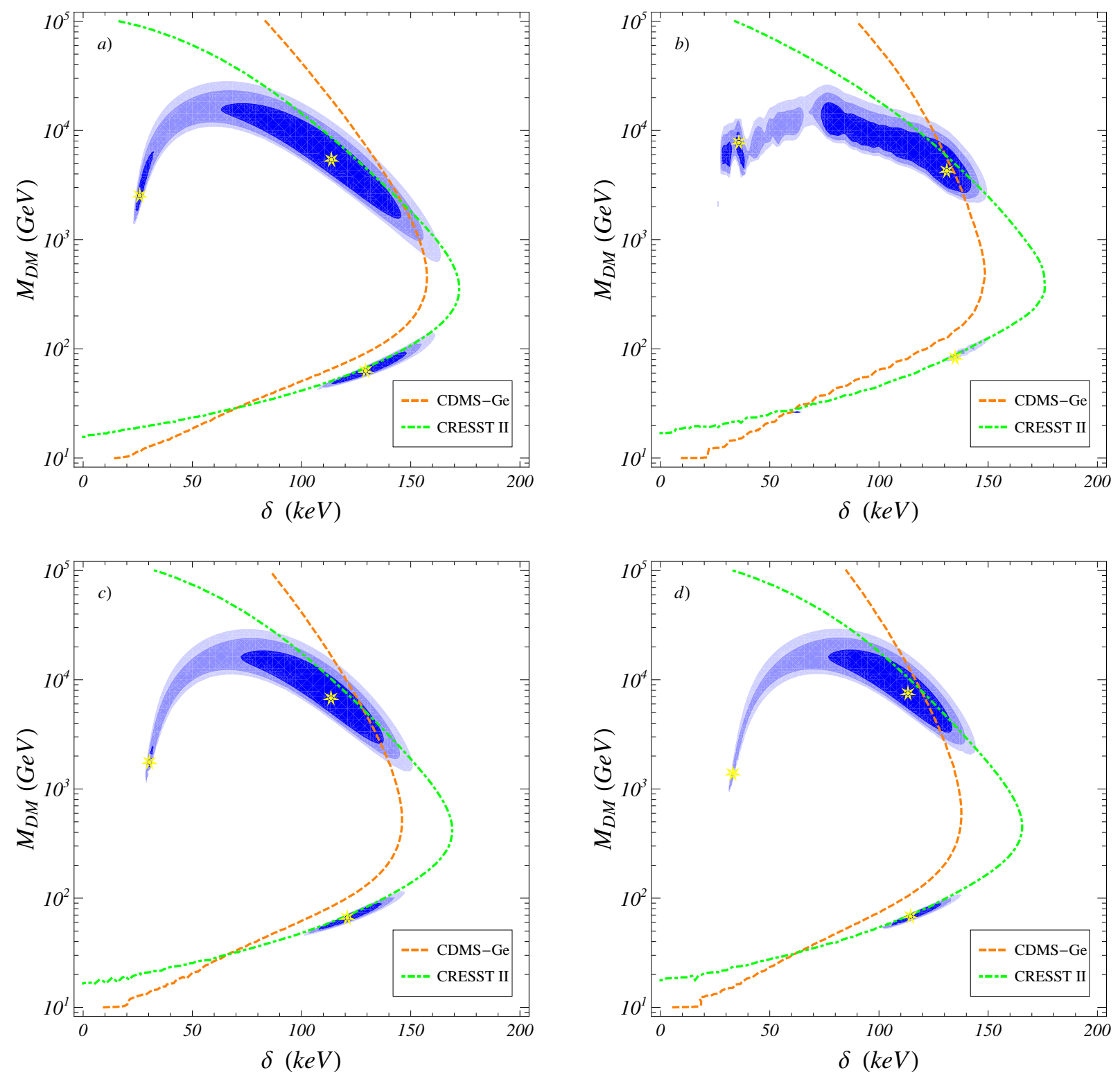

Figure 15: Inelastic Scenario, allowed regions in the plane $\delta-M_{D M}$

a) Standard Maxwellian halo $\left(\rho_{D M}=0.3 \mathrm{GeV} / \mathrm{cm}^{3}, v_{c}=220 \mathrm{~km} / \mathrm{s}, v_{0}=220 \mathrm{~km} / \mathrm{s}, v_{\text {esc }}=600 \mathrm{~km} / \mathrm{s}\right)$

b) $N$-body simulation with baryons $\left(\rho_{D M}=0.37 \mathrm{GeV} / \mathrm{cm}^{3}, v_{c}=220 \mathrm{~km} / \mathrm{s}\right)$

c) Generalized Maxwellian halo $\left(\rho_{D M}=0.37 \mathrm{GeV} / \mathrm{cm}^{3}, v_{c}=190 \mathrm{~km} / \mathrm{s}, v_{0}=301 \mathrm{~km} / \mathrm{s}, \alpha=1.5\right.$ )

d) Generalized Maxwellian halo $\left(\rho_{D M}=0.37 \mathrm{GeV} / \mathrm{cm}^{3}, v_{c}=190 \mathrm{~km} / \mathrm{s}, v_{0}=332 \mathrm{~km} / \mathrm{s}, \alpha=1.95\right.$ )

For c) and d), the circular velocity $v_{c}$ has been reduced compared to the standard value in order to mimic a halo rotation. DAMA contours correspond to 90, 99 and $99.9 \%$ CL. Stars indicate local best-fit points. All other exclusion curves are at the $99.9 \%$ CL. 